\title{
Partitions and Sylvester waves
}

\author{
Cormac O'Sullivan*
}

July 15,2017

\begin{abstract}
The restricted partition function $p_{N}(n)$ counts the partitions of the integer $n$ into at most $N$ parts. In the nineteenth century Sylvester described these partitions as a sum of waves. We give detailed descriptions of these waves and, for the first time, show the asymptotics of the initial waves as $N$ and $n$ both go to infinity at about the same rate. This allows us to see when the initial waves are a good approximation to $p_{N}(n)$ in this situation. Our proofs employ the saddle-point method of Perron and the dilogarithm.
\end{abstract}

\section{Introduction}

\subsection{Decomposing partitions into waves}

Let $p(n)$ be the number of partitions of the integer $n$. This is the number of ways to write $n$ as a sum of non-increasing positive integers. Also let $p_{N}(n)$ count the partitions of $n$ with at most $N$ summands. (We are following the notation that was convenient in [Rad73, O'S15, O'S16a].) As usual, $p(0)$ and $p_{N}(0)$ are defined to be 1. Since the work of Cayley [Cay56] and Sylvester [Syl82], we know that

$$
p_{N}(n)=\sum_{k=1}^{N} W_{k}(N, n) \quad\left(N \in \mathbb{Z}_{\geqslant 1}, n \in \mathbb{Z}_{\geqslant 0}\right)
$$

where each $W_{k}(N, n)$ may be expressed in terms of a sequence of $k$ polynomials $w_{k, m}(N, x) \in \mathbb{Q}[x]$ for $0 \leqslant m \leqslant k-1$. Similarly to [SZ12, p. 641] we write

$$
W_{k}(N, n)=\left[w_{k, 0}(N, n), w_{k, 1}(N, n), \ldots, w_{k, k-1}(N, n)\right],
$$

where the notation in (1.2) indicates that the value of $W_{k}(N, n)$ is given by one of the polynomials on the right and we select $w_{k, j}(N, n)$ when $n \equiv j \bmod k$. As we will see, the degrees of the polynomials on the right of (1.2) are at most $\lfloor N / k\rfloor-1$.

For example, with $N=5$ we have $p_{5}(n)=W_{1}(5, n)+\cdots+W_{5}(5, n)$ where

$$
\begin{aligned}
& W_{1}(5, n)=\left[30 n^{4}+900 n^{3}+9300 n^{2}+38250 n+50651\right] / 86400, \\
& W_{2}(5, n)=[2 n+15,-2 n-15] / 128, \\
& W_{3}(5, n)=[2,-1,-1] / 27, \\
& W_{4}(5, n)=[1,1,-1,-1] / 16, \\
& W_{5}(5, n)=[4,-1,-1,-1,-1] / 25 .
\end{aligned}
$$

2010 Mathematics Subject Classification. 11P82, 41A60

Key words and phrases. Restricted partitions, Sylvester waves, asymptotics, saddle-point method.

This research was supported, in part, by a grant of computer time from the City University of New York High Performance Computing Center under NSF Grants CNS-0855217, CNS-0958379 and ACI-1126113. Support for this project was also provided by a PSC-CUNY Award, jointly funded by The Professional Staff Congress and The City University of New York. 
This computation was first carried out by Cayley in 1856, [Cay56, p. 132]. Sylvester called $W_{k}(N, n)$ the $k$-th wave and provided the formula

$$
W_{k}(N, n)=\operatorname{Res}_{z=0} \sum_{\rho} \frac{\rho^{n} e^{n z}}{\left(1-\rho^{-1} e^{-z}\right)\left(1-\rho^{-2} e^{-2 z}\right) \cdots\left(1-\rho^{-N} e^{-N z}\right)}
$$

in [Syl82]. Here $\operatorname{Res}_{z=0}$ indicates the coefficient of $1 / z$ in the Laurent expansion about 0 , and the sum is over all primitive $k$-th roots of unity $\rho$. For example, Glaisher shows in [Gla09, Sections 19-30] that the first wave is

$$
W_{1}(N, n)=\frac{(-1)^{N-1}}{N !} \sum_{j_{0}+j_{1}+\cdots+j_{N}=N-1}(-n)^{j_{0}} B_{j_{1}} \cdots B_{j_{N}} \frac{1^{j_{1}} \cdots N^{j_{N}}}{j_{0} ! j_{1} ! \cdots j_{N} !}
$$

where the Bernoulli numbers $B_{m}$ are the constant terms of the Bernoulli polynomials $B_{m}(t)$, defined with

$$
\frac{z e^{t z}}{e^{z}-1}=\sum_{m=0}^{\infty} B_{m}(t) \frac{z^{m}}{m !} .
$$

We will take (1.4) as our definition of $W_{k}(N, n)$ and prove in Section 2 that (1.1) and (1.2) follow.

Clearly the first wave $W_{1}(5, n)$ will make the largest contribution to $p_{5}(n)$ for large $n$. Similarly, for any fixed $N$ as $n \rightarrow \infty$ we have $p_{N}(n) \sim W_{1}(N, n)$, since the first wave has the biggest degree, and the well-known asymptotid 1

$$
p_{N}(n)=\frac{n^{N-1}}{(N-1) ! N !}+O\left(n^{N-2}\right)
$$

then follows from $(1.5)$.

\subsection{Main results}

A more difficult question is how $W_{1}(N, n)$ or the first waves $W_{1}(N, n)+W_{2}(N, n)+\cdots$ compare with $p_{N}(n)$ as $N$ and $n$ both go to $\infty$ together. Suppose, to begin with, that $N=n$. We obtain the unrestricted partitions $p(n)=p_{n}(n)$, and see for example that

$$
\begin{aligned}
22=p(8) & =W_{1}(8,8)+W_{2}(8,8)+W_{3}(8,8)+\cdots+W_{8}(8,8) \\
& \approx 21.4127+0.4112-0.0566+\cdots+0.0625 .
\end{aligned}
$$

So $W_{1}(n, n)$ gives a good approximation to $p(n)$ for $n=8$ and we may ask if $W_{1}(n, n) \sim p(n)$ for large $n$.

To examine this question we recall the formula of Hardy, Ramanujan and Rademacher, given for example in $[\operatorname{Rad} 73$, Eq. (120.10)] as

$$
p(n)=\sum_{k=1}^{\infty} \frac{A_{k}(n)}{2 \sqrt{3 k}(n-1 / 24)}\left(\cosh \left(\frac{2 \pi}{\sqrt{6} k} \sqrt{n-1 / 24}\right)-\frac{\sinh \left(\frac{2 \pi}{\sqrt{6} k} \sqrt{n-1 / 24}\right)}{\frac{2 \pi}{\sqrt{6} k} \sqrt{n-1 / 24}}\right)
$$

where $A_{k}(n)$ may be expressed with Selberg's formula [Rad73, Eq. (123.2)] as

$$
A_{k}(n)=\sqrt{k / 3} \sum_{\substack{0 \leqslant \ell \leqslant 2 k-1 \\\left(3 \ell^{2}-\ell\right) / 2 \equiv-n \bmod k}}(-1)^{\ell} \cos \left(\frac{(6 \ell-1) \pi}{6 k}\right) .
$$

The $k=1$ term of (1.8) is already a good approximation to $p(n)$; using Rademacher's bound in [Rad73, $\mathrm{p}$. 277] for the error after truncation yields, since $A_{1}(n)=1$,

$$
p(n)=\frac{1}{4 \sqrt{3}(n-1 / 24)} \exp \left(\frac{2 \pi}{\sqrt{6}} \sqrt{n-1 / 24}\right)\left(1+O\left(\frac{1}{\sqrt{n}}\right)\right) .
$$

\footnotetext{
${ }^{1}$ As usual, the notation $f(z)=O(g(z))$, or equivalently $f(z) \ll g(z)$, means that there exists a $C$ so that $|f(z)| \leqslant C \cdot g(z)$ for all $z$ in a specified range. The number $C$ is called the implied constant.
} 
The only comparison between $W_{1}(n, n)$ and $p(n)$ we have found in the literature is by Szekeres. He compared the expansion of $p(n)$ as a sum of Sylvester waves to the expansion (1.8), noting in [Sze51, $\mathrm{p}$. 108] that the corresponding terms seem to match up for small values of $n$. For example, when $n=8$ the first three terms of (1.8) give

$$
p(8) \approx 21.7092+0.3463-0.0896+\cdots .
$$

He speculated that the correspondence between (1.7) and (1.10) would improve as $n$ gets larger.

In fact we show that the sum of the first waves $W_{1}(n, n)+W_{2}(n, n)+\cdots$ does not stay close to $p(n)$ for large $n$. The size of these waves is approximately $\exp (0.068 n) / n^{2}$ whereas $p(n)$ grows like $\exp (2.565 \sqrt{n}) / n$ according to (1.9). More precisely, for the first 100 waves $W_{1}(n, n)+\cdots+W_{100}(n, n)$, we prove the following.

Theorem 1.1. There exist explicit constants $w_{0}$ and $z_{0}$ in $\mathbb{C}$ so that as $n \rightarrow \infty$

$$
\sum_{k=1}^{100} W_{k}(n, n)=\operatorname{Re}\left[\left(2 z_{0} e^{-3 \pi i z_{0}}\right) \frac{w_{0}^{-n}}{n^{2}}\right]+O\left(\frac{\left|w_{0}\right|^{-n}}{n^{3}}\right) .
$$

The numbers $w_{0}$ and $z_{0}$ are coming from the saddle-point method, with $w_{0}$ the unique solution in $\mathbb{C}$ to

$$
\operatorname{Li}_{2}(w)-2 \pi i \log (w)=0,
$$

where $\mathrm{Li}_{2}(w)$ denotes the dilogarithm function, as described in Section 4.2, and $z_{0}:=1+\log \left(1-w_{0}\right) /(2 \pi i)$ so that

$$
w_{0}=1-e^{2 \pi i z_{0}}, \quad 1 / 2<\operatorname{Re}\left(z_{0}\right)<3 / 2 .
$$

In [O'S16c] it is shown that $w_{0}$ and $z_{0}$ may be found to any precision and we have

$$
w_{0} \approx 0.916198-0.182459 i, \quad z_{0} \approx 1.181475+0.255528 i .
$$

Hence we may equivalently present (1.11) as

$$
\sum_{k=1}^{100} W_{k}(n, n)=\frac{e^{U n}}{n^{2}}\left(\psi_{1} \cdot \sin \left(\tau_{1}+V n\right)+O\left(\frac{1}{n}\right)\right)
$$

with

$$
\begin{array}{rlrl}
U & :=-\log \left|w_{0}\right| \approx 0.0680762, & V:=\arg \left(1 / w_{0}\right) \approx 0.196576, \\
\psi_{1}:=\left|2 i \cdot z_{0} e^{-3 \pi i z_{0}}\right| \approx 26.8713, & \tau_{1}:=\arg \left(2 i \cdot z_{0} e^{-3 \pi i z_{0}}\right) \approx-3.06816 .
\end{array}
$$

For large $n$ we see that the first waves trace an oscillating sine wave with amplitude growing exponentially. The period of the oscillation is $2 \pi / V \approx 31.96311$ and successive waves increase by a factor of approximately $e^{U} \approx 1.07045$. The number $w_{0}$, which is controlling this behaviour, is in fact a zero of the dilogarithm on a non-principal branch and was first identified in [O'S15]. The Rademacher coefficients studied in [O'S15] have very similar asymptotic properties to Sylvester waves, and indeed they may be expressed in terms of each other - see [O’S15, Sect. 4].

\begin{tabular}{c|c|c|c|c}
$n$ & $W_{1}(n, n)$ & $W_{2}(n, n)$ & $W_{3}(n, n)$ & $W_{4}(n, n)$ \\
\hline 1000 & $2.41 \times 10^{31}$ & $4.09 \times 10^{13}$ & $-3.03 \times 10^{7}$ & $8.14 \times 10^{4}$ \\
1500 & $2.32 \times 10^{39}$ & $2.40 \times 10^{17}$ & $2.49 \times 10^{10}$ & $5.52 \times 10^{6}$ \\
2000 & $4.37 \times 10^{53}$ & $4.98 \times 10^{23}$ & $-8.22 \times 10^{13}$ & $6.98 \times 10^{8}$
\end{tabular}

Table 1: Relative sizes of the first waves

Theorem 1.1 is not the best possible result; we expect it to be true with the sum of the first 100 waves replaced by just the first wave. Numerically, the other waves are much smaller than the first, as shown in Table 1 for example. 


\begin{tabular}{c|c|c|c}
$n$ & $p(n)$ & $W_{1}(n, n)$ & $\operatorname{Re}\left[\left(2 z_{0} e^{-3 \pi i z_{0}}\right) \frac{w_{0}^{-n}}{n^{2}}\right]$ \\
\hline 1200 & $\mathbf{4 . 6 2} \times \mathbf{1 0}^{\mathbf{3 4}}$ & $\mathbf{4 . 6 2} \times \mathbf{1 0}^{\mathbf{3 4}}$ & $1.90 \times 10^{30}$ \\
1300 & $\mathbf{1 . 6 1} \times \mathbf{1 0}^{\mathbf{3 6}}$ & $\mathbf{1 . 6 1} \times \mathbf{1 0}^{\mathbf{3 6}}$ & $3.95 \times 10^{33}$ \\
1400 & $\mathbf{4 . 9 0} \times \mathbf{1 0}^{\mathbf{3 7}}$ & $\mathbf{5 . 2 1} \times \mathbf{1 0}^{\mathbf{3 7}}$ & $3.12 \times 10^{36}$ \\
1500 & $1.33 \times 10^{39}$ & $2.32 \times 10^{39}$ & $9.67 \times 10^{38}$ \\
1600 & $3.24 \times 10^{40}$ & $-\mathbf{8 . 0 8} \times \mathbf{1 0}^{\mathbf{4 1}}$ & $-\mathbf{8 . 9 3} \times \mathbf{1 0}^{\mathbf{4 1}}$ \\
1700 & $7.18 \times 10^{41}$ & $-\mathbf{1 . 5 7} \times \mathbf{1 0}^{\mathbf{4 5}}$ & $-\mathbf{1 . 6 0} \times \mathbf{1 0}^{\mathbf{4 5}}$ \\
1800 & $1.46 \times 10^{43}$ & $-\mathbf{1 . 2 0} \times \mathbf{1 0}^{\mathbf{4 8}}$ & $-\mathbf{1 . 2 1} \times \mathbf{1 0}^{\mathbf{4 8}}$
\end{tabular}

Table 2: Comparing $p(n), W_{1}(n, n)$ and the asymptotics from Theorem 1.1

Comparing (1.14) and (1.9), we see that the first waves must eventually become much larger than $p(n)$. In fact $\psi_{1} e^{U n} / n^{2}$ equals the right side of $(1.9)$ for $n \approx 1480$. We see in Table 2 that $p(n)$ and $W_{1}(n, n)$ get further and further apart after this value. On the other hand, for $n \ll 1480$ the first waves are a good approximation to $p(n)$ as we see in Section 8.2 ,

Our main result is the following, of which Theorem 1.1 is a special case. Instead of restricting to $n=N$ we allow $n$ in a range between $-\lambda^{+} N$ and $\lambda^{+} N$.

Theorem 1.2. Let $\lambda^{+}$be a positive real number. Suppose $N \in \mathbb{Z}_{\geqslant 1}$ and $\lambda N \in \mathbb{Z}$ for $\lambda$ satisfying $|\lambda| \leqslant \lambda^{+}$. Then there are explicit coefficients $a_{0}(\lambda), a_{1}(\lambda), \ldots$ so that

$$
\sum_{k=1}^{100} W_{k}(N, \lambda N)=\operatorname{Re}\left[\frac{w_{0}^{-N}}{N^{2}}\left(a_{0}(\lambda)+\frac{a_{1}(\lambda)}{N}+\cdots+\frac{a_{m-1}(\lambda)}{N^{m-1}}\right)\right]+O\left(\frac{\left|w_{0}\right|^{-N}}{N^{m+2}}\right)
$$

as $N \rightarrow \infty$ where $a_{0}(\lambda)=2 z_{0} e^{-\pi i z_{0}(1+2 \lambda)}$ and the implied constant depends only on $\lambda^{+}$and $m \geqslant 0$.

The formula for the next coefficient, $a_{1}(\lambda)$, is given in Proposition 5.2. These first waves show the same basic oscillating behavior as the $\lambda=1$ case, with period $2 \pi / V \approx 31.96311$ and increasing by a factor of approximately $e^{U} \approx 1.07045$ with each $N$ :

$$
\sum_{k=1}^{100} W_{k}(N, \lambda N)=\frac{e^{U N}}{N^{2}} \psi_{\lambda} \cdot \sin \left(\tau_{\lambda}+V N\right)+O\left(\frac{e^{U N}}{N^{3}}\right)
$$

where, consistent with (1.16),

$$
\psi_{\lambda}:=2\left|z_{0}\right| e^{\pi \operatorname{Im}\left(z_{0}\right)(1+2 \lambda)}, \quad \tau_{\lambda}:=\arg \left(i z_{0}\right)-\pi \operatorname{Re}\left(z_{0}\right)(1+2 \lambda) .
$$

As before, we expect Theorem 1.2 to be true with the left side of (1.17) replaced by just the first wave $W_{1}(N, \lambda N)$. Instances of Theorem 1.2, comparing the right side of (1.17) to the first wave, are displayed in Table 3 .

\begin{tabular}{cc|cccc|c}
$N$ & $\lambda$ & $m=1$ & $m=2$ & $m=3$ & $m=5$ & $W_{1}(N, \lambda N)$ \\
\hline 3300 & $1 / 3$ & $-8.35526 \times 10^{90}$ & $-8.22612 \times 10^{90}$ & $-8.22662 \times 10^{90}$ & $-8.22663 \times 10^{90}$ & $-8.22663 \times 10^{90}$ \\
3300 & 1 & $-9.05354 \times 10^{91}$ & $-8.97235 \times 10^{91}$ & $-8.97192 \times 10^{91}$ & $-8.97194 \times 10^{91}$ & $-8.97194 \times 10^{91}$ \\
3300 & 2 & $-2.02861 \times 10^{92}$ & $-1.88676 \times 10^{92}$ & $-1.89108 \times 10^{92}$ & $-1.89104 \times 10^{92}$ & $-1.89104 \times 10^{92}$
\end{tabular}

Table 3: The approximations of Theorem 1.2 to $W_{1}(N, \lambda N)$.

Taking $m=1$ and $\lambda=r / N$ in Theorem 1.2 easily gives the following corollary.

Corollary 1.3. Fix $r \in \mathbb{Z}$. Then

$$
\sum_{k=1}^{100} W_{k}(N, r)=\operatorname{Re}\left[\left(2 z_{0} e^{-\pi i z_{0}}\right) \frac{w_{0}^{-N}}{N^{2}}\right]+O\left(\frac{\left|w_{0}\right|^{-N}}{N^{3}}\right)
$$

as $N \rightarrow \infty$ for an implied constant depending only on $r$. 
Thus we see that even in the wave expansion of $P_{N}(1)=1$, corresponding to $r=1$ in (1.20), the first waves are becoming exponentially large with $N$. So in general we expect some of the individual waves on the right of (1.1) to be much larger than $p_{N}(n)$, indicating a lot of cancelation on the right side.

A situation where the first waves are the same size as $p_{N}(n)$ is given next.

Theorem 1.4. Let $\lambda$ be in the range $0.2 \leqslant \lambda \leqslant 2.9$. Then for positive integers $N$ and $\lambda N^{2}$ we have

$$
p_{N}\left(\lambda N^{2}\right)=\left(\sum_{k=1}^{100} W_{k}\left(N, \lambda N^{2}\right)\right)\left(1+O\left(e^{-0.1 N}\right)\right)
$$

as $N \rightarrow \infty$ where the implied constant is absolute.

Theorem 1.4 is easily shown using the methods from the proof of Theorem 1.2. However, a stronger result that replaces the 100 waves by the first wave and increases the range of $\lambda$ should be possible. Indeed the work in [Sze51] suggests that the correct range for $\lambda$ should be much larger.

\section{Sylvester waves}

\subsection{Basic properties}

As in [O'S16a, Sect. 2.1] we define

$$
Q(z ; N, \sigma):=\frac{e^{2 \pi i \sigma z}}{\left(1-e^{2 \pi i 1 z}\right)\left(1-e^{2 \pi i 2 z}\right) \cdots\left(1-e^{2 \pi i N z}\right)}
$$

and it is easy to show that for any $\sigma \in \mathbb{C}$

$$
\begin{aligned}
Q(z+1 ; N, \sigma) & =e^{2 \pi i \sigma} Q(z ; N, \sigma), \\
Q(-z ; N, \sigma) & =\overline{Q(\bar{z} ; N, \bar{\sigma})}, \\
Q(-z ; N, \sigma) & =(-1)^{N} Q(z ; N, N(N+1) / 2-\sigma) .
\end{aligned}
$$

Our goal is to express the Sylvester waves as residues of $Q(z ; N, \sigma)$. As a function of $z, Q(z ; N, \sigma)$ is meromorphic with all poles contained in $\mathbb{Q}$. More precisely, the set of poles of $Q(z ; N, \sigma)$ in $[0,1)$ equals $\mathscr{F}_{N}$, the Farey fractions of order $N$ in $[0,1)$. We write

$$
Q_{h k \sigma}(N):=2 \pi i \operatorname{Res}_{z=h / k} Q(z ; N, \sigma)
$$

for $h / k \in \mathscr{F}_{N}$. When dealing with residues the next simple relations for $c \in \mathbb{C}$ are useful:

$$
\operatorname{Res}_{z=0} f(z)=c \operatorname{Res}_{z=0} f(c z), \quad \operatorname{Res}_{z=0} f(z+c)=\operatorname{Res}_{z=c} f(z), \quad \overline{\operatorname{Res}_{z=c} f(z)}=\operatorname{Res}_{z=\bar{c}} \overline{f(\bar{z})} .
$$

With (2.2), (2.3) and (2.6) we obtain

$$
\begin{aligned}
Q_{01 \sigma}(N) & =\overline{Q_{01 \bar{\sigma}}(N)}, \\
Q_{(k-h) k \sigma}(N) & =e^{2 \pi i \sigma} \overline{Q_{h k \bar{\sigma}}(N)} \quad(k \geqslant 2) .
\end{aligned}
$$

A simple exercise with (2.6) also shows, for $h / k \in \mathscr{F}_{N}, \rho=e^{2 \pi i h / k}$ and $\sigma \in \mathbb{C}$, that

$$
\operatorname{Res}_{z=0} \frac{e^{2 \pi i \sigma h / k} e^{\sigma z}}{\left(1-\rho^{-1} e^{-z}\right)\left(1-\rho^{-2} e^{-2 z}\right) \cdots\left(1-\rho^{-N} e^{-N z}\right)}=-\overline{Q_{h k(-\sigma)}(N)} .
$$

From (2.7), 2.8) and (2.9) we find:

Proposition 2.1. For all $k, N \in \mathbb{Z}_{\geqslant 1}$ and all $n \in \mathbb{Z}$,

$$
W_{k}(N, n)=-\sum_{0 \leqslant h<k,(h, k)=1} Q_{h k(-n)}(N) .
$$


As shown in [O'S16a, Thm. 2.1], we may relate the right side of (2.10) above to the restricted partitions. Briefly, the generating function

$$
\sum_{n=0}^{\infty} p_{N}(n) q^{n}=\prod_{j=1}^{N} \frac{1}{1-q^{j}}
$$

with $q$ replaced by $e^{2 \pi i z}$ becomes $Q(z ; N, 0)$. We therefore have

$$
\int_{w}^{w+1} Q(z ; N,-n) d z=\left\{\begin{array}{lll}
p_{N}(n) & \text { if } & n \in \mathbb{Z}_{\geqslant 0} \\
0 & \text { if } & n \in \mathbb{Z}_{<0}
\end{array}\right.
$$

for any $w \in \mathbb{C}$ with $\operatorname{Im}(w)$ large enough. Integrating $Q(z ; N,-n)$ around the rectangle with corners $w+1$, $w, \bar{w}$ and $\overline{w+1}$, using (2.2), (2.4) and Cauchy's residue theorem proves:

Theorem 2.2. For $N \in \mathbb{Z}_{\geqslant 1}$ and $n \in \mathbb{Z}$ we have

$$
-\sum_{h / k \in \mathscr{F}_{N}} Q_{h k(-n)}(N)= \begin{cases}p_{N}(n) & \text { if } \quad 0 \leqslant n \\ 0 & \text { if } \quad-N(N+1) / 2<n<0 \\ (-1)^{N+1} p_{N}(-n-N(N+1) / 2) & \text { if } n \leqslant-N(N+1) / 2 .\end{cases}
$$

With Proposition 2.1 and Theorem 2.2 we have shown that $p_{N}(n)=\sum_{k=1}^{N} W_{k}(N, n)$, (i.e. equality (1.1), follows from the wave definition (1.4). This result is known as Sylvester's Theorem - see for example [O'S15, Sect.4] and its contained references. The next result, showing that (1.2) is also a consequence of (1.4), follows for example from [Gla09, Sections 4-7] which uses partial fractions. We give a new proof.

Proposition 2.3. For each wave $W_{k}(N, n)$, equation $(1.2)$ is valid for polynomials $w_{k, m}(N, x) \in \mathbb{Q}[x]$ that have degree at most $\lfloor N / k\rfloor-1$. They satisfy

$$
w_{k, 0}(N, x)+w_{k, 1}(N, x)+\cdots+w_{k, k-1}(N, x)=0 \quad(k \geqslant 2)
$$

and more generally, with any factorization $k=b c$ and $0 \leqslant \ell \leqslant b-1$,

$$
w_{k, \ell}(N, x)+w_{k, \ell+b}(N, x)+w_{k, \ell+2 b}(N, x)+\cdots+w_{k, \ell+(c-1) b}(N, x)=0 \quad(c \geqslant 2) .
$$

Proof. Following Apostol in [Apo51, Eq. (3.1)], write

$$
\frac{z}{\rho e^{z}-1}=\sum_{m=0}^{\infty} \beta_{m}(\rho) \frac{z^{m}}{m !} \quad(\rho \in \mathbb{C}) .
$$

Then (1.4) may be expressed as

$$
\begin{gathered}
W_{k}(N, n)=\frac{(-1)^{N-1}}{N !} \sum_{\rho} \rho^{-n}\left[\text { coeff. of } z^{N-1}\right] e^{-n z}\left(\frac{z}{\rho e^{z}-1}\right)\left(\frac{2 z}{\rho^{2} e^{2 z}-1}\right) \cdots\left(\frac{N z}{\rho^{N} e^{N z}-1}\right) \\
=\frac{(-1)^{N-1}}{N !} \sum_{j=0}^{N-1} \frac{(-n)^{j}}{j !} \sum_{j_{1}+j_{2}+\cdots+j_{N}=N-1-j} \frac{1^{j_{1}} 2^{j_{2}} \cdots N^{j_{N}}}{j_{1} ! j_{2} ! \cdots j_{N} !}\left(\sum_{\rho} \rho^{-n} \beta_{j_{1}}(\rho) \beta_{j_{2}}\left(\rho^{2}\right) \cdots \beta_{j_{N}}\left(\rho^{N}\right)\right)
\end{gathered}
$$

where $\rho$ is summed over all primitive $k$ th roots of unity. It is clear from the form of (2.16) that $W_{k}(N, n)$ is a polynomial in $n$ where, for fixed $k$ and $N$, the polynomial depends only on $n \bmod k$. Hence

$$
\begin{aligned}
w_{k, m}(N, x)=\frac{(-1)^{N-1}}{N !} \sum_{j=0}^{N-1} \frac{(-x)^{j}}{j !} & \\
& \times \sum_{j_{1}+j_{2}+\cdots+j_{N}=N-1-j} \frac{1^{j_{1}} 2^{j_{2}} \cdots N^{j_{N}}}{j_{1} ! j_{2} ! \cdots j_{N} !}\left(\sum_{\rho} \rho^{-m} \beta_{j_{1}}(\rho) \beta_{j_{2}}\left(\rho^{2}\right) \cdots \beta_{j_{N}}\left(\rho^{N}\right)\right) .
\end{aligned}
$$


The inner sum in (2.17) will be zero if $j_{1}+\cdots+j_{N}$ is too small since $\beta_{0}(w)=0$ for $w \neq 1$. For $w=1$ we have $\beta_{0}(1)=1$. Therefore the smallest value of $j_{1}+\cdots+j_{N}$ that gives a possible nonzero value for the inner sum is $N-\lfloor N / k\rfloor$. Hence we may assume $j \leqslant\lfloor N / k\rfloor-1$ in (2.17), proving the bound for the degree.

We have $\beta_{m}(1)=B_{m}$ and, by a formula of Glaisher [Gla09, Sect. 97] (see also [O'S15, Prop. 3.2]),

$$
\beta_{m}(\xi)=(-1)^{m-1} m \sum_{j=1}^{m}\left\{\begin{array}{c}
m \\
j
\end{array}\right\} \frac{(j-1) !}{(\xi-1)^{j}} \quad(\xi \neq 1)
$$

where $\left\{\begin{array}{c}m \\ j\end{array}\right\}$ is the Stirling number that denotes the number of ways to partition a set of size $m$ into $j$ nonempty subsets. For fixed $j_{1}, \ldots, j_{N}$ the value of the inner sum in $(2.17)$ is therefore in the field $\mathbb{Q}(\rho)$. This value is unchanged under any automorphism of $\mathbb{Q}(\rho)$, since the primitive $k$ th roots will just be permuted. It follows that this value is in the fixed field of all automorphisms and so rational. Finally, (2.13) and (2.14) follow from (2.17) and the identity

$$
\rho^{\ell}\left(1+\rho^{b}+\rho^{2 b}+\cdots+\rho^{(c-1) b}\right)=0 \quad(c \geqslant 2) .
$$

\subsection{Explicit waves}

The formula 2.17) gives a convenient expression for the $k$ th wave, especially when combined with the result from [O’S15, Eq. (3.6)]:

$$
\beta_{m}(\xi)=k^{m-1} \sum_{j=0}^{k-1} \xi^{j} B_{m}(j / k)
$$

for all $m \in \mathbb{Z}_{\geqslant 0}$ and all $\xi \in \mathbb{C}$ with $\xi^{k}=1$ for $k \in \mathbb{Z}_{\geqslant 1}$. Sylvester in [Syl82] and Glaisher in [Gla09] developed different descriptions for $W_{k}(N, n)$. For some details of this and further treatments see Dowker's papers [Dowa, Dowb] and also [BGK01, FR02]. In [RF06, Eq. (46)] the waves are written in terms of Bernoulli and Eulerian polynomials of higher order. An interesting expression for the first wave $W_{1}$ that does not involve Bernoulli polynomials has recently appeared in [DV17].

For fixed $k$ and $0 \leqslant w \leqslant k-1$ set

$$
s_{m, w}(N):=\sum_{1 \leqslant j \leqslant N, j \equiv w \bmod k} j^{m} .
$$

A variation of a result of Glaisher using the Apostol coefficients (2.15) is the following, given in [O'S15, Eq. (4.8)].

Theorem 2.4. For $k, N \in \mathbb{Z}_{\geqslant 1}, s:=\lfloor N / k\rfloor$ and $n \in \mathbb{Z}$

$$
\begin{aligned}
W_{k}(N, n) & =\sum_{\rho} \frac{(-1)^{s-1} \rho^{-n}}{k^{2 s} \cdot s ! \prod_{1 \leqslant r \leqslant N-k s}\left(1-\rho^{r}\right)} \\
\times & \sum_{1 j_{1}+2 j_{2}+\cdots+N j_{N}=s-1} \frac{1}{j_{1} ! j_{2} ! \cdots j_{N} !}\left(-n-\frac{N(N+1)}{2}-\sum_{w=0}^{k-1} \frac{\beta_{1}\left(\rho^{w}\right) \cdot s_{1, w}(N)}{1 \cdot 1 !}\right)^{j_{1}} \\
& \quad \times\left(-\sum_{w=0}^{k-1} \frac{\beta_{2}\left(\rho^{w}\right) \cdot s_{2, w}(N)}{2 \cdot 2 !}\right)^{j_{2}} \cdots\left(-\sum_{w=0}^{k-1} \frac{\beta_{N}\left(\rho^{w}\right) \cdot s_{N, w}(N)}{N \cdot N !}\right)^{j_{N}}
\end{aligned}
$$

where the outer sum is over all primitive $k$-th roots of unity $\rho$.

This means of calculating $W_{k}(N, n)$ is computationally faster than (2.17) when $N$ is large and the wave computations in Tables 1, 2, 3, 9 and 10 were carried out using (2.20). An efficient means of computing waves that avoids roots of unity is given in [SZ12].

For a simple example,

$$
W_{1}(6, n)=\left(12 n^{5}+630 n^{4}+12320 n^{3}+110250 n^{2}+439810 n+598731\right) / 1036800 .
$$


We notice that all the coefficients of $W_{1}(6, n)$ are positive (the same was true for $W_{1}(5, n)$ in (1.3) ) and this positivity continues in $W_{1}(N, n)$ for increasing $N$. However, it must eventually fail; as we see from Table 2 , at least one of the coefficients of the polynomial $W_{1}(1600, n)$ is negative.

For $k=2$ we have $W_{2}(N, n)=(-1)^{n} w_{2,0}(N, n)$ by (2.13). In this case $\beta_{j}(-1)=\left(2^{j}-1\right) B_{j}$ and we find, for instance with $N=10$,

$$
W_{2}(10, n)=(-1)^{n}\left(30 n^{4}+3300 n^{3}+125400 n^{2}+1905750 n+9406331\right) / 88473600 .
$$

The third wave for $N=10$ is

$$
W_{3}(10, n)=\left[6 n^{2}+344 n+4317,-28 n-770,-6 n^{2}-316 n-3547\right] / 52488 .
$$

For large values of $k$ we may simplify the formula (2.20). When $N / 2<k \leqslant N$ we have $s=1$ and hence

$$
W_{k}(N, n)=k^{-2} \sum_{\rho} \frac{\rho^{-n}}{\left(1-\rho^{1}\right)\left(1-\rho^{2}\right) \cdots\left(1-\rho^{N-k}\right)} .
$$

As we saw in Proposition 2.3, (2.21) must be a rational number and for fixed $k$ and $N$ it depends only on $n$ mod $k$. We next show how these rationals may be expressed more explicitly. Note also the similarity of (2.21) with the Fourier-Dedekind sums of [BDR02].

In the simplest case $k=N$ of 2.21)

$$
W_{N}(N, n)=N^{-2} \sum_{\rho} \rho^{-n}
$$

which was already given in [RF06, Eq. (47)]. The sum in (2.22) is over all primitive $N$-th roots of unity. Such sums are called Ramanujan sums and may be evaluated, see [HW08, Thm. 271], as the integer

$$
c_{N}(n):=\sum_{\rho} \rho^{n}=\sum_{d \mid(N, n)} d \cdot \mu(N / d)
$$

where $\mu(m)$ is the Möbius function, defined to be 0 unless $m$ is squarefree and otherwise -1 to the power of the number of prime factors of $m$ (with $\mu(1)=1$ ). The next result gives formulas for $W_{k}(N, n)$ explicitly in terms of rationals when $k$ is $N, N-1$ or $N-2$. Recall the Bernoulli polynomials $B_{m}(x)$ from (1.6) with $B_{1}(x)=x-1 / 2$ and $B_{2}(x)=x^{2}-x+1 / 6$.

Proposition 2.5. For $n \in \mathbb{Z}$

$$
\begin{aligned}
W_{k}(k, n) & =\frac{1}{k^{2}} c_{k}(n) \quad\left(k \in \mathbb{Z}_{\geqslant 1}\right), \\
W_{k}(k+1, n) & =\frac{-1}{k^{2}} \sum_{j=0}^{k-1} B_{1}\left(\frac{j}{k}\right) c_{k}(j-n) \quad\left(k \in \mathbb{Z}_{\geqslant 2}\right), \\
W_{k}(k+2, n) & =\frac{-1}{4 k^{2}} \sum_{j=0}^{k-1}\left[3 B_{1}\left(\frac{j}{k}\right)+k \cdot B_{2}\left(\frac{j}{k}\right)\right] c_{k}(j-n)-\frac{(-1)^{n}}{4 k^{2}} \sum_{j=0}^{\ell-1} B_{1}\left(\frac{j}{\ell}\right) c_{\ell}(j-n)
\end{aligned}
$$

where in (2.25) we have $k \in \mathbb{Z}_{\geqslant 3}$ and

$$
\ell:= \begin{cases}k & \text { if } \quad k \equiv 0 \bmod 4 \\ k / 2 & \text { if } \quad k \equiv 2 \bmod 4 \\ 2 k & \text { if } \quad k \equiv 1,3 \bmod 4 .\end{cases}
$$

Proof. We have already seen (2.23). With $m=1$ in (2.18) and (2.19) we find

$$
\frac{1}{1-\rho}=-\beta_{1}(\rho)=-\sum_{j=0}^{k-1} B_{1}(j / k) \rho^{j}
$$


for $\rho$ any $k$-th root of unity with $\rho \neq 1$. Summing over all primitive $k$-th root of unity we have

$$
W_{k}(k+1, n)=\frac{1}{k^{2}} \sum_{\rho} \frac{\rho^{-n}}{1-\rho}=\frac{-1}{k^{2}} \sum_{j=0}^{k-1} B_{1}(j / k) \sum_{\rho} \rho^{j-n}
$$

and (2.24) follows. For $k \geqslant 3$ we have

$$
W_{k}(k+2, n)=\frac{1}{k^{2}} \sum_{\rho} \frac{\rho^{-n}}{(1-\rho)\left(1-\rho^{2}\right)}=\frac{-1}{k^{2}} \sum_{\rho}\left[\frac{1}{4} \frac{\rho^{-n}}{1-\rho}+\frac{1}{4} \frac{\rho^{-n}}{1+\rho}+\frac{1}{2} \frac{\rho^{-n}}{(1-\rho)^{2}}\right]
$$

using the partial fraction decomposition for $1 /\left((1-\rho)\left(1-\rho^{2}\right)\right)$ as in [O'S15, p. 735]. The first sum on the right of (2.27) has been found with (2.26). The second sum may be evaluated in the same way since

$$
\sum_{\rho} \frac{\rho^{-n}}{1+\rho}=(-1)^{n} \sum_{\rho} \frac{(-\rho)^{-n}}{1-(-\rho)}
$$

and $\rho$ is a primitive $k$-th root of unity if and only if $-\rho$ is a primitive $\ell$-th root of unity. The third sum on the right of (2.27) can be found similarly. Use $m=2$ in (2.19) and (2.18) to get

$$
\frac{1}{(1-\rho)^{2}}-\frac{1}{1-\rho}=\frac{-\beta_{2}(\rho)}{2}=\frac{-k}{2} \sum_{j=0}^{k-1} B_{2}(j / k) \rho^{j}
$$

It should be possible to find similar formulas for $W_{k}(k+3, n), W_{k}(k+4, n)$ etc. When $k$ is a prime $p$, Proposition 2.5 implies

$$
\begin{aligned}
W_{p}(p, n) & =[p-1,-1,-1, \cdots,-1] / p^{2}, \\
W_{p}(p+1, n) & =\left[p^{\prime}, p^{\prime}-1, p^{\prime}-2, \cdots, p^{\prime}-p+1\right] / p^{2}
\end{aligned}
$$

in the notation (1.2) and for $p^{\prime}:=(p-1) / 2$. The identity (2.28) appears in [Cay56, p. 131]. Also

$$
W_{p}(p+2, n)=\frac{1}{4 p^{2}}\left(-\bar{n}^{2}+\bar{n}(p-3)+\frac{(-1)^{\bar{n}} p}{2}-\frac{p^{2}-9 p+11}{6}\right)
$$

for $p \geqslant 3$, where $n \equiv \bar{n} \bmod p$ and $0 \leqslant \bar{n} \leqslant p-1$.

\subsection{General denumerants}

The results in Section 2.1 may be extended in a straightforward manner to the general restricted partition problem considered by Cayley and Sylvester. Let $A=\left\{a_{1}, a_{2}, \cdots, a_{N}\right\}$ be a fixed set of positive integers, not necessarily distinct, and write $p_{A}(n)$ for the number of solutions to

$$
a_{1} x_{1}+a_{2} x_{2}+\cdots+a_{N} x_{N}=n \quad\left(x_{1}, x_{2}, \ldots, x_{N} \in \mathbb{Z}_{\geqslant 0}\right) .
$$

Our focus, $p_{N}(n)$, equals $p_{A}(n)$ for $A=\{1,2, \cdots, N\}$. In general for $n \in \mathbb{Z}_{\geqslant 0}$,

$$
p_{A}(n)=\sum_{k \mid \text { some } a_{i}} W_{k}(A, n)
$$

where

$$
W_{k}(A, n)=\left[w_{k, 0}(A, n), w_{k, 1}(A, n), \ldots, w_{k, k-1}(A, n)\right],
$$

and, with a sum over all primitive $k$-th roots of unity $\rho$ as before,

$$
W_{k}(A, n)=\operatorname{Res}_{z=0} \sum_{\rho} \frac{\rho^{n} e^{n z}}{\left(1-\rho^{-a_{1}} e^{-a_{1} z}\right)\left(1-\rho^{-a_{2}} e^{-a_{2} z}\right) \cdots\left(1-\rho^{-a_{N}} e^{-a_{N} z}\right)} .
$$

Proposition 2.6. For each wave $W_{k}(A, n)$, equation (2.32) is valid for polynomials $w_{k, j}(A, x) \in \mathbb{Q}[x]$ that have degree at most one less than the number of elements of $A$ that are divisible by $k$. With any factorization $k=b c$ and $0 \leqslant \ell \leqslant b-1$, they satisfy

$$
w_{k, \ell}(A, x)+w_{k, \ell+b}(A, x)+w_{k, \ell+2 b}(A, x)+\cdots+w_{k, \ell+(c-1) b}(A, x)=0 \quad(c \geqslant 2) .
$$




\section{Asymptotics for Sylvester waves}

For $N \in \mathbb{Z}_{\geqslant 1}$ and $n \in \mathbb{Z}$, Theorem 2.2 implies

$$
p_{N}(n)=-\sum_{h / k \in \mathscr{F}_{N}} Q_{h k(-n)}(N),
$$

where we define $p_{N}(n)$ to be 0 for $-N(N+1) / 2<n<0$ and $(-1)^{N+1} p_{N}(-n-N(N+1) / 2)$ if $n \leqslant-N(N+1) / 2$. Put

$$
\mathcal{A}(N):=\{h / k: N / 2<k \leqslant N, h=1 \text { or } h=k-1\} \subseteq \mathscr{F}_{N}
$$

and for $N$ large we partition $\mathscr{F}_{N}$ into three parts: $\mathscr{F}_{100}, \mathcal{A}(N)$ and the rest. The sum (3.1) becomes

$$
p_{N}(n)=-\left[\sum_{h / k \in \mathscr{F}_{100}}+\sum_{h / k \in \mathscr{F}_{N}-\left(\mathscr{F}_{100} \cup \mathcal{A}(N)\right)}+\sum_{h / k \in \mathcal{A}(N)}\right] Q_{h k(-n)}(N) .
$$

The sum over $\mathscr{F}_{100}$ is the sum of the first 100 waves

$$
\sum_{k=1}^{100} W_{k}(N, n)=-\sum_{h / k \in \mathscr{F}_{100}} Q_{h k(-n)}(N) .
$$

Write the sum over $\mathcal{A}(N)$, see [O'S16a, Eq. (1.21)], as

$$
\mathcal{A}_{1}(N,-n):=\sum_{h / k \in \mathcal{A}(N)} Q_{h k(-n)}(N)
$$

With (3.3), (3.4) and (3.5) we may write our key identity as

$$
\sum_{k=1}^{100} W_{k}(N, n)=p_{N}(n)+\mathcal{A}_{1}(N,-n)+\sum_{h / k \in \mathscr{F}_{N}-\left(\mathscr{F}_{100} \cup \mathcal{A}(N)\right)} Q_{h k(-n)}(N) .
$$

Since every $Q_{h k(-n)}(N)$ in (3.5) is the residue of a simple pole, they may be calculated as in [O'S16a, Eq. (1.22)] to obtain

$$
\mathcal{A}_{1}(N,-n)=\operatorname{Im} \sum_{\frac{N}{2}<k \leqslant N} \frac{2(-1)^{k}}{k^{2}} \exp \left(\frac{i \pi}{2}\left[\frac{-N^{2}-N-4 n}{k}+3 N\right]\right) \prod_{N-k}^{-1}(1 / k)
$$

where we used the reciprocal of the product

$$
\prod_{m}(\theta):=\prod_{j=1}^{m} 2 \sin (\pi j \theta)
$$

with $\prod_{0}(\theta):=1$. The right side of (3.7) can be analyzed in great detail and its asymptotics found:

Theorem 3.1. Let $\lambda^{+}$be a positive real number. Suppose $N \in \mathbb{Z}_{\geqslant 1}$ and $\lambda N \in \mathbb{Z}$ for $\lambda$ satisfying $|\lambda| \leqslant \lambda^{+}$. Then for $a_{0}(\lambda)=2 z_{0} e^{-\pi i z_{0}(1+2 \lambda)}$ and explicit $a_{1}(\lambda), a_{2}(\lambda), \ldots$ we have

$$
\mathcal{A}_{1}(N,-\lambda N)=\operatorname{Re}\left[\frac{w_{0}^{-N}}{N^{2}}\left(a_{0}(\lambda)+\frac{a_{1}(\lambda)}{N}+\cdots+\frac{a_{m-1}(\lambda)}{N^{m-1}}\right)\right]+O\left(\frac{\left|w_{0}\right|^{-N}}{N^{m+2}}\right)
$$

for an implied constant depending only on $\lambda^{+}$and $m$. 
6.

This theorem is proved in Section 5. The next result, on the last component of (3.6), is shown in Section

Theorem 3.2. Let $\lambda^{+}$be a positive real number. Suppose $N \in \mathbb{Z}_{\geqslant 1}$ and $\lambda N \in \mathbb{Z}$ for $\lambda$ satisfying $|\lambda| \leqslant \lambda^{+}$. Then for an implied constant depending only on $\lambda^{+}$,

$$
\sum_{h / k \in \mathscr{F}_{N}-\left(\mathscr{F}_{100} \cup \mathcal{A}(N)\right)} Q_{h k(-\lambda N)}(N)=O\left(e^{0.055 N}\right) .
$$

These two results, combined with bounds for $p_{N}(n)$, imply Theorem 1.2 ;

Proof of Theorem 1.2 Combining (3.6) with Theorems 3.1, 3.2 shows

$$
\begin{aligned}
\sum_{k=1}^{100} W_{k}(N, \lambda N) & =p_{N}(\lambda N)+\mathcal{A}_{1}(N,-\lambda N)+O\left(e^{0.055 N}\right) \\
& =p_{N}(\lambda N)+\operatorname{Re}\left[\frac{w_{0}^{-N}}{N^{2}}\left(a_{0}(\lambda)+\frac{a_{1}(\lambda)}{N}+\cdots+\frac{a_{m-1}(\lambda)}{N^{m-1}}\right)\right]+O\left(\frac{\left|w_{0}\right|^{-N}}{N^{m+2}}\right)
\end{aligned}
$$

where $\left|w_{0}\right|^{-N} \approx e^{0.068 N}$. The estimate

$$
p_{N}(\lambda N) \ll e^{(2 \pi \sqrt{|\lambda| / 6}) \sqrt{N}}
$$

follows from $\left|p_{N}(n)\right| \leqslant p_{N}(|n|) \leqslant p(|n|)$ and (1.9). Calculus shows the crude bound $e^{r \sqrt{N}} \leqslant e^{r^{2} /(4 t)} \cdot e^{t N}$ for any $r \geqslant 0, t>0$. Hence, with $r=2 \pi \sqrt{|\lambda| / 6}$ and $t=0.055$, say,

$$
p_{N}(\lambda N) \ll e^{(2 \pi \sqrt{|\lambda| / 6}) \sqrt{N}} \leqslant e^{\pi^{2}|\lambda| /(6(0.055))} e^{0.055 N} .
$$

Therefore $p_{N}(\lambda N)$ may be included in the error term in (3.11). This completes the proof of Theorem 1.2 .

\section{Required results}

\subsection{The saddle-point method}

We will apply Perron's saddle-point method from [Per17] in Sections 5] and 7, The exact form we need is given in $\left[\overline{\mathrm{O}^{\prime} \mathrm{S}}\right]$ and requires the following discussion to state it precisely.

The usual convention that the principal branch of $\log$ has arguments in $(-\pi, \pi]$ is used. As in (4.3) below, powers of nonzero complex numbers take the corresponding principal value $z^{\tau}:=e^{\tau \log (z)}$ for $\tau \in \mathbb{C}$.

Our contours of integration $\mathcal{C}$ will lie in a bounded region of $\mathbb{C}$ and be parameterized by a continuous function $c:[0,1] \rightarrow \mathbb{C}$ that has a continuous derivative except at a finite number of points. For any appropriate $f$, integration along the corresponding contour $\mathcal{C}$ is defined as $\int_{\mathcal{C}} f(z) d z:=\int_{0}^{1} f(c(t)) c^{\prime}(t) d t$ in the normal way.

We make the following assumptions and definitions.

Assumptions 4.1. We have $\mathcal{B}$ a neighborhood of $z_{0} \in \mathbb{C}$. Let $\mathcal{C}$ be a contour as described above, with $z_{0}$ a point on it. Suppose $p(z)$ and $q(z)$ are holomorphic functions on a domain containing $\mathcal{B} \cup \mathcal{C}$. We assume $p(z)$ is not constant and hence there must exist $\mu \in \mathbb{Z}_{\geqslant 1}$ and $p_{0} \in \mathbb{C}_{\neq 0}$ so that

$$
p(z)=p\left(z_{0}\right)+p_{0}\left(z-z_{0}\right)^{\mu}(1-\phi(z)) \quad(z \in \mathcal{B})
$$

with $\phi$ holomorphic on $\mathcal{B}$ and $\phi\left(z_{0}\right)=0$. Let $\omega_{0}:=\arg \left(p_{0}\right)$ and we will need the steepest-descent angles

$$
\theta_{\ell}:=-\frac{\omega_{0}}{\mu}+\frac{2 \pi \ell}{\mu} \quad(\ell \in \mathbb{Z}) .
$$

We also assume that $\mathcal{B}, \mathcal{C}, p(z), q(z)$ and $z_{0}$ are independent of $N>0$. Finally, let $K(q)$ be a bound for $|q(z)|$ on $\mathcal{B} \cup \mathcal{C}$. 
Theorem 4.2 (The saddle-point method of Perron). Suppose Assumptions 4.1 hold and $\mu$ is even. Let $\mathcal{C}$ be a contour beginning at $z_{1}$, passing through $z_{0}$ and ending at $z_{2}$, with these points all distinct. Suppose that

$$
\operatorname{Re}(p(z))>\operatorname{Re}\left(p\left(z_{0}\right)\right) \text { for all } z \in \mathcal{C}, z \neq z_{0} .
$$

Let $\mathcal{C}$ approach $z_{0}$ in a sector of angular width $2 \pi / \mu$ about $z_{0}$ with bisecting angle $\theta_{k} \pm \pi$, and initially leave $z_{0}$ in a sector of the same size with bisecting angle $\theta_{k}$. Then for every $M \in \mathbb{Z}_{\geqslant 0}$,

$$
\int_{\mathcal{C}} e^{-N \cdot p(z)} q(z) d z=e^{-N \cdot p\left(z_{0}\right)}\left(\sum_{m=0}^{M-1} \Gamma\left(\frac{2 m+1}{\mu}\right) \frac{2 \alpha_{2 m}(q) \cdot e^{2 \pi i k(2 m+1) / \mu}}{N^{(2 m+1) / \mu}}+O\left(\frac{K(q)}{N^{(2 M+1) / \mu}}\right)\right)
$$

as $N \rightarrow \infty$ where the implied constant is independent of $N$ and $q$. The numbers $\alpha_{s}(q)$ are given by

$$
\alpha_{s}(q)=\frac{1}{\mu \cdot s !} p_{0}^{-(s+1) / \mu} \frac{d^{s}}{d z^{s}}\left\{q(z) \cdot(1-\phi(z))^{-(s+1) / \mu}\right\}_{z=z_{0}} .
$$

Theorem 4.2 is proved as Corollary 5.1 in $[\overline{\mathrm{O}} \mathrm{S}]$ with the innovation of making the error independent of $q$. Note that [O'S] has $p(z)$ with the opposite sign but all other notation, e.g. $p_{0}$, is the same. The numbers $\alpha_{s}(q)$ depend on $p$ and $z_{0}$, but we have highlighted the dependence on $q$ since we will be applying Theorem 4.2 with $q$ varying. Another description of $\alpha_{s}(q)$ may be given in terms of the power series for $p$ and $q$ near $z_{0}$ :

$$
p(z)-p\left(z_{0}\right)=\sum_{s=0}^{\infty} p_{s}\left(z-z_{0}\right)^{s+\mu}, \quad q(z)=\sum_{s=0}^{\infty} q_{s}\left(z-z_{0}\right)^{s} .
$$

This requires the partial ordinary Bell polynomials, [Com74, p. 136], which are defined with the generating function

$$
\left(p_{1} x+p_{2} x^{2}+p_{3} x^{3}+\cdots\right)^{j}=\sum_{i=j}^{\infty} \hat{B}_{i, j}\left(p_{1}, p_{2}, p_{3}, \ldots\right) x^{i}
$$

Clearly $\hat{B}_{i, 0}\left(p_{1}, p_{2}, p_{3}, \ldots\right)$ is 1 for $i=0$ and is 0 for $i \geqslant 1$. Also

$$
\hat{B}_{i, j}\left(p_{1}, p_{2}, p_{3}, \ldots\right)=\sum_{n_{1}+n_{2}+\cdots+n_{j}=i} p_{n_{1}} p_{n_{2}} \cdots p_{n_{j}}
$$

for $j \geqslant 1$ from [CFW87, p. 156] where the sum is over all possible $n_{1}, n_{2}, \cdots \in \mathbb{Z}_{\geqslant 1}$.

Proposition 4.3. For $\alpha_{s}(q)$ defined in (4.3),

$$
\alpha_{s}(q)=\frac{1}{\mu} p_{0}^{-(s+1) / \mu} \sum_{i=0}^{s} q_{s-i} \sum_{j=0}^{i}\left(\begin{array}{c}
-(s+1) / \mu \\
j
\end{array}\right) \hat{B}_{i, j}\left(\frac{p_{1}}{p_{0}}, \frac{p_{2}}{p_{0}}, \cdots\right) .
$$

Proposition 4.3 is due to Campbell, Fröman and Walles [CFW87, pp. 156-158]. The above formulation is proved in [O'S, Prop. 7.2]. Wojdylo also rediscovered this formula; see the references in [O'S]. The following result is [O'S Prop. 7.3].

Proposition 4.4. With Assumptions 4.1 and $\alpha_{s}(q)$ defined in (4.3),

$$
\alpha_{s}(q)=O\left(K^{*}(q) \cdot C^{s}\right) \quad \text { for } \quad s \in \mathbb{Z}_{\geqslant 0}
$$

where $K^{*}(q)$ is a bound for $|q(z)|$ on $\mathcal{B}$. The positive constant $C$ and the implied constant in (4.8) are both independent of $q$ and $s$. 


\subsection{The dilogarithm}

As described in [Max03], [Zag07], [O’S16c] for example, the dilogarithm is initially defined as

$$
\operatorname{Li}_{2}(z):=\sum_{n=1}^{\infty} \frac{z^{n}}{n^{2}} \quad \text { for }|z| \leqslant 1
$$

with an analytic continuation given by $-\int_{0}^{z} \log (1-u) / u d u$. This makes the dilogarithm a multi-valued holomorphic function with a branch points at $1, \infty$ (and off the principal branch another branch point at 0 ). We let $\operatorname{Li}_{2}(z)$ denote the dilogarithm on its principal branch so that $\operatorname{Li}_{2}(z)$ is a single-valued holomorphic function on $\mathbb{C}-[1, \infty)$.

We may describe $\operatorname{Li}_{2}(z)$ for $z$ on the unit circle as

$$
\begin{array}{ll}
\operatorname{Re}\left(\operatorname{Li}_{2}\left(e^{2 \pi i x}\right)\right)=\sum_{n=1}^{\infty} \frac{\cos (2 \pi n x)}{n^{2}}=\pi^{2} B_{2}(x-\lfloor x\rfloor) & (x \in \mathbb{R}), \\
\operatorname{Im}\left(\operatorname{Li}_{2}\left(e^{2 \pi i x}\right)\right)=\sum_{n=1}^{\infty} \frac{\sin (2 \pi n x)}{n^{2}}=\mathrm{Cl}_{2}(2 \pi x) & (x \in \mathbb{R})
\end{array}
$$

where $B_{2}(x):=x^{2}-x+1 / 6$ is the second Bernoulli polynomial and

$$
\mathrm{Cl}_{2}(\theta):=-\int_{0}^{\theta} \log |2 \sin (x / 2)| d x \quad(\theta \in \mathbb{R})
$$

is Clausen's integral. Note that $\mathrm{Li}_{2}(1)=\zeta(2)=\pi^{2} / 6$. The graph of $\mathrm{Cl}_{2}(\theta)$ resembles a slanted sine wave see [O'S16c, Fig. 1], for example. Combine (4.10) and (4.11) to get, for $z \in \mathbb{R}$ with $m \leqslant z \leqslant m+1, m \in \mathbb{Z}$,

$$
\mathrm{Cl}_{2}(2 \pi z)=-i \operatorname{Li}_{2}\left(e^{2 \pi i z}\right)+i \pi^{2}\left(z^{2}-(2 m+1) z+m^{2}+m+1 / 6\right) .
$$

Then the right of (4.13) gives the continuation of $\mathrm{Cl}_{2}(2 \pi z)$ to $z \in \mathbb{C}$ with $m<\operatorname{Re}(z)<m+1$.

As $z$ crosses the branch cuts the dilogarithm enters new branches. From [Max03, Sect. 3], the value of the analytically continued dilogarithm is always given by

$$
\mathrm{Li}_{2}(z)+4 \pi^{2} A+2 \pi i B \log (z)
$$

for some $A, B \in \mathbb{Z}$.

The saddle-points we need in our asymptotic calculations are closely related to zeros of the analytically continued dilogarithm and in [O'S16c] we have made a study of its zeros on every branch. When the continued dilogarithm takes the form (4.14) with $B=0$, there will be a zero if and only if $A \geqslant 0$ and, for each such $A$, the zero will be unique and lie on the real line. The cases we will require have $B \neq 0$. In these cases there are no real zeros so we may avoid the branch cuts and look for solutions to

$$
\mathrm{Li}_{2}(z)+4 \pi^{2} A+2 \pi i B \log (z)=0 \quad(z \in \mathbb{C}, z \notin(-\infty, 0] \cup[1, \infty), A, B \in \mathbb{Z}) .
$$

The next result is shown in Theorems 1.1 and 1.3 of [O'S16c].

Theorem 4.5. For nonzero $B \in \mathbb{Z}$, (4.15) has solutions if and only if $-|B| / 2<A \leqslant|B| / 2$. For such a pair $A, B$ the solution $z$ is unique. This unique solution, $w(A, B)$, may be found to arbitrary precision using Newton's method.

By conjugating (4.15) it is clear that

$$
w(A,-B)=\overline{w(A, B)} .
$$

So for nonzero $B$ the first zeros are $w(0,1)$ and its conjugate $w(0,-1)$. We have

$$
w(0,-1) \approx 0.9161978162-0.1824588972 i
$$


and this zero was denoted by $w_{0}$ in Section 1.2 . We will also need

$$
\begin{aligned}
& w(0,-2) \approx 0.9684820460-0.1095311065 i \\
& w(1,-3) \approx-0.4594734813-0.8485350380 i .
\end{aligned}
$$

Define

$$
p_{d}(z):=\frac{-\mathrm{Li}_{2}\left(e^{2 \pi i z}\right)+\mathrm{Li}_{2}(1)+4 \pi^{2} d}{2 \pi i z},
$$

a single-valued holomorphic function away from the vertical branch cuts $(-i \infty, n]$ for $n \in \mathbb{Z}$. Its first derivatives are

$$
\begin{aligned}
& p_{d}^{\prime}(z)=-\frac{1}{z}\left(p_{d}(z)-\log \left(1-e^{2 \pi i z}\right)\right), \\
& p_{d}^{\prime \prime}(z)=-\frac{1}{z}\left(2 p_{d}^{\prime}(z)+\frac{2 \pi i \cdot e^{2 \pi i z}}{1-e^{2 \pi i z}}\right) .
\end{aligned}
$$

The last result in this section is [O'S16a, Thm. 2.4] and identifies the saddle-points we will need.

Theorem 4.6. Fix integers $m$ and $d$ with $-|m| / 2<d \leqslant|m| / 2$. Then there is a unique solution to $p_{d}^{\prime}(z)=0$ for $z \in \mathbb{C}$ with $m-1 / 2<\operatorname{Re}(z)<m+1 / 2$ and $z \notin(-i \infty, m]$. Denoting this solution by $z^{*}$, it is given by

$$
z^{*}=m+\log (1-w(d,-m)) /(2 \pi i)
$$

and satisfies

$$
p_{d}\left(z^{*}\right)=\log (w(d,-m))
$$

\section{Proof of Theorem 3.1}

Proof of Theorem 3.1 We require a modification of the proof of [O'S16a, Thm 1.6]. The following should be read alongside Sections 2-5 of [O'S16a] where there are more details. First define

$$
g_{\ell}(z):=-\frac{B_{2 \ell}}{(2 \ell) !}(\pi z)^{2 \ell-1} \cot ^{(2 \ell-2)}(\pi z) .
$$

We approximate the reciprocal of the sine product $\prod_{m}(\theta)$, as defined in (3.8), with [O'S16a, Thm. 4.1], based on Euler-Maclaurin summation. A special case, see [O'S16a, Cor. 4.2], shows

$$
\prod_{N-k}^{-1}(1 / k)=O\left(e^{0.05 N}\right) \quad \text { for } \quad \frac{N}{k} \in[1,1.01] \cup[1.49,2)
$$

and

$$
\begin{aligned}
& \prod_{N-k}^{-1}(1 / k)=\left(\frac{N / k}{2 N \sin (\pi(N / k-1))}\right)^{1 / 2} \exp \left(\frac{N}{2 \pi N / k} \mathrm{Cl}_{2}(2 \pi N / k)\right) \\
& \quad \times \exp \left(\sum_{\ell=1}^{L-1} \frac{g_{\ell}(N / k)}{N^{2 \ell-1}}\right)+O\left(e^{0.05 N}\right) \quad \text { for } \quad \frac{N}{k} \in(1.01,1.49)
\end{aligned}
$$

with $L=\lfloor 0.006 \pi e \cdot N\rfloor$. The implied constants in (5.2), (5.3) are absolute. We may combine (3.7), (5.2) and (5.3) by first making the following definitions:

$$
\begin{aligned}
p(z) & :=\frac{\operatorname{Li}_{2}(1)-\mathrm{Li}_{2}\left(e^{2 \pi i z}\right)}{2 \pi i z}, \\
q(z) & :=\left(\frac{z}{2 \sin (\pi(z-1))}\right)^{1 / 2} \exp (-\pi i z / 2), \\
v(z ; N, \sigma) & :=\frac{2 \pi i \sigma z}{N}+\sum_{\ell=1}^{L-1} \frac{g_{\ell}(z)}{N^{2 \ell-1}}, \quad(L=\lfloor 0.006 \pi e \cdot N\rfloor) .
\end{aligned}
$$


We used (4.13) with $m=1$ to convert Clausen's integral into the dilogarithm in (5.4). (Note that all the occurrences of the function $p$ in this section refer to (5.4) and not the partition function.) Then with $\hat{z}=\hat{z}(N, k):=N / k$ define

$$
\mathcal{A}_{2}(N, \sigma):=\frac{2}{N^{1 / 2}} \operatorname{Im} \sum_{k: \hat{z} \in(1.01,1.49)} \frac{(-1)^{k}}{k^{2}} \exp (-N(p(\hat{z})-\pi i / \hat{z})) q(\hat{z}) \exp (v(\hat{z} ; N, \sigma))
$$

where the index notation means we are summing over all integers $k$ such that $1.01<N / k<1.49$. It follows, as in [O'S16a, Eq. (4.11)], that for any $\sigma \in \mathbb{Z}$ and an absolute implied constant we have

$$
\mathcal{A}_{2}(N, \sigma)=\mathcal{A}_{1}(N, \sigma)+O\left(e^{0.05 N}\right) .
$$

When $\sigma=-\lambda N$ we may remove the $\exp (-2 \pi i \lambda z)$ factor from $\exp (v(z ; N, \sigma))$ and include it in the new function

$$
f_{\mathcal{A}, \lambda}(z):=q(z) \exp (-2 \pi i \lambda z)
$$

to get

$$
\mathcal{A}_{2}(N,-\lambda N)=\frac{2}{N^{1 / 2}} \operatorname{Im} \sum_{k: \hat{z} \in(1.01,1.49)} \frac{(-1)^{k}}{k^{2}} \exp (-N(p(\hat{z})-\pi i / \hat{z})) f_{\mathcal{A}, \lambda}(\hat{z}) \exp (v(\hat{z} ; N, 0)) .
$$

It is shown in [O'S16a, Prop. 4.7] that $q(z)$ and $\exp (v(z ; N, 0))$ are holomorphic and absolutely bounded on a domain containing the box $\mathbb{B}_{1}$ for

$$
\mathbb{B}_{m}:=\{z \in \mathbb{C}: m+0.01 \leqslant \operatorname{Re}(z) \leqslant m+0.49,-1 \leqslant \operatorname{Im}(z) \leqslant 1\} .
$$

Since $|\exp (-2 \pi i \lambda z)| \leqslant \exp \left(\lambda^{+} 2 \pi|z|\right)$ it follows that

$$
f_{\mathcal{A}, \lambda}(z) \exp (v(z ; N, 0)) \ll 1 \quad \text { for } \quad z \in \mathbb{B}_{1}
$$

with an implied constant depending only on $\lambda^{+}$. The proof of [O'S16a, Thm. 4.3] now goes through unchanged, except that we have $\sigma=0$ and $f_{\mathcal{A}, \lambda}$ instead of $q$. This theorem allows us to replace the sum (5.10) by the integral

$$
\mathcal{A}_{3}(N,-\lambda N):=\frac{2}{N^{3 / 2}} \operatorname{Im} \int_{1.01}^{1.49} e^{-N \cdot p(z)} f_{\mathcal{A}, \lambda}(z) \cdot \exp (v(z ; N, 0)) d z
$$

where, for an implied constant depending only on $\lambda^{+}$(coming from the bound (5.12)),

$$
\mathcal{A}_{3}(N,-\lambda N)=\mathcal{A}_{2}(N,-\lambda N)+O\left(e^{0.05 N}\right) .
$$

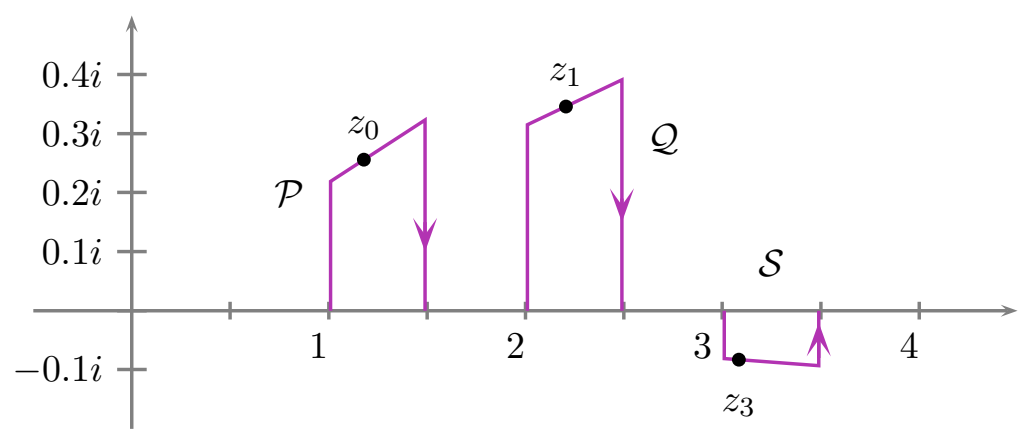

Figure 1: The paths of integration $\mathcal{P}, \mathcal{Q}$ and $\mathcal{S}$

The form of (5.13) allows us to find its asymptotic expansion using the saddle-point method as was done in [O'S16a]. We have seen in Theorem 4.6 that $p^{\prime}(z)=0$ has a unique solution for $0.5<\operatorname{Re}(z)<1.5$ 
given by $z=1+\log (1-w(0,-1)) /(2 \pi i)$. (The function $p(z)$ is the case $d=0$ of $p_{d}(z)$.) In the notation of (1.12) we write $w_{0}=w(0,-1)$ and $z_{0}$ is the saddle-point $1+\log \left(1-w_{0}\right) /(2 \pi i)$.

In the notation of Assumptions 4.1 and Theorem 4.2, we find $\mu=2, p_{0} \approx 0.504-0.241 i$ and the steepest-descent angles are $\theta_{0} \approx 0.223$ and $\theta_{1}=\pi+\theta_{0}$. Let $c:=1+i \operatorname{Im}\left(z_{0}\right) / \operatorname{Re}\left(z_{0}\right)$. We move the path of integration in (5.13) to the path $\mathcal{P}$ consisting of the straight line segments joining the points 1.01, $1.01 c, 1.49 c$ and 1.49. This path passes through $z_{0}$ as shown in Figure 1. Since the integrand in (5.13) is holomorphic on a domain containing $\mathbb{B}_{1}$, Cauchy's theorem ensures that the integral remains the same under this change of path. It is proved in [O'S16a, Thm. 5.2] that

$$
\operatorname{Re}\left(p(z)-p\left(z_{0}\right)\right)>0 \quad \text { for all } \quad z \in \mathcal{P}, z \neq z_{0} .
$$

Recall from (1.15) and (4.20) that

$$
e^{-p\left(z_{0}\right)}=w_{0}^{-1} \quad \text { and } \quad e^{-\operatorname{Re}\left(p\left(z_{0}\right)\right)}=\left|w_{0}\right|^{-1} \approx e^{0.068} .
$$

To apply the saddle-point method we state one further result, which is [O'S16a, Prop. 5.8]. Set $u_{0,0}:=1$ and for $j \in \mathbb{Z}_{\geqslant 1}$ put

$$
u_{0, j}(z):=\sum_{m_{1}+3 m_{2}+5 m_{3}+\cdots=j} \frac{g_{1}(z)^{m_{1}}}{m_{1} !} \frac{g_{2}(z)^{m_{2}}}{m_{2} !} \cdots \frac{g_{j}(z)^{m_{j}}}{m_{j} !} .
$$

Proposition 5.1. There are functions $u_{0, j}(z)$ (defined above) and $\zeta_{d}(z ; N, 0)$ which are holomorphic on a domain containing the box $\mathbb{B}_{1}$ and have the following property. For all $z \in \mathbb{B}_{1}$,

$$
\exp (v(z ; N, 0))=\sum_{j=0}^{d-1} \frac{u_{0, j}(z)}{N^{j}}+\zeta_{d}(z ; N, 0) \quad \text { for } \quad \zeta_{d}(z ; N, 0)=O\left(\frac{1}{N^{d}}\right)
$$

with an implied constant depending only on $d$ where $1 \leqslant d \leqslant 2 L-1$ and $L=\lfloor 0.006 \pi e \cdot N\rfloor$.

Proposition 5.1 implies

$$
\begin{aligned}
\mathcal{A}_{3}(N,-\lambda N)=\operatorname{Im}\left[\sum_{j=0}^{d-1} \frac{2}{N^{3 / 2+j}} \int_{\mathcal{P}} e^{-N \cdot p(z)} \cdot f_{\mathcal{A}, \lambda}(z) \cdot u_{0, j}(z) d z\right. \\
\left.\quad+\frac{2}{N^{3 / 2}} \int_{\mathcal{P}} e^{-N \cdot p(z)} \cdot f_{\mathcal{A}, \lambda}(z) \cdot \zeta_{d}(z ; N, 0) d z\right]+O\left(e^{0.06 N}\right)
\end{aligned}
$$

where, by (5.12), (5.15) and Proposition 5.1, the last term in the parentheses in (5.18) is

$$
\ll \frac{1}{N^{3 / 2}} \int_{\mathcal{P}}\left|e^{-N \cdot p(z)}\right| \cdot 1 \cdot \frac{1}{N^{d}} d z \ll \frac{1}{N^{d+3 / 2}} e^{-N \operatorname{Re}\left(p\left(z_{0}\right)\right)}=\frac{\left|w_{0}\right|^{-N}}{N^{d+3 / 2}},
$$

for an implied constant depending only on $\lambda^{+}$. Applying Theorem 4.2 to each integral in the first part of (5.18) we obtain, since $k=0$,

$$
\begin{aligned}
& \int_{\mathcal{P}} e^{-N \cdot p(z)} \cdot f_{\mathcal{A}, \lambda}(z) \cdot u_{0, j}(z) d z \\
&=e^{-N \cdot p\left(z_{0}\right)}\left(\sum_{m=0}^{M-1} \Gamma\left(m+\frac{1}{2}\right) \frac{2 \alpha_{2 m}\left(f_{\mathcal{A}, \lambda} \cdot u_{0, j}\right)}{N^{m+1 / 2}}+O\left(\frac{K\left(f_{\mathcal{A}, \lambda} \cdot u_{0, j}\right)}{N^{M+1 / 2}}\right)\right) .
\end{aligned}
$$


The error term in (5.19) corresponds to an error in (5.18) of size $O\left(\left|w_{0}\right|^{-N} / N^{M+j+2}\right)$. Choose $M=d$ so that this error is less than $O\left(\left|w_{0}\right|^{-N} / N^{d+3 / 2}\right)$ for all $j \geqslant 0$. Therefore

$$
\begin{array}{r}
\mathcal{A}_{3}(N,-\lambda N)=\operatorname{Im}\left[\sum_{j=0}^{d-1} \frac{4}{N^{j+3 / 2}} e^{-N \cdot p\left(z_{0}\right)} \sum_{m=0}^{d-1} \Gamma\left(m+\frac{1}{2}\right) \frac{\alpha_{2 m}\left(f_{\mathcal{A}, \lambda} \cdot u_{0, j}\right)}{N^{m+1 / 2}}\right]+O\left(\frac{\left|w_{0}\right|^{-N}}{N^{d+3 / 2}}\right) \\
=\operatorname{Im}\left[w_{0}^{-N} \sum_{t=0}^{2 d-2} \frac{4}{N^{t+2}} \sum_{m=\max (0, t-d+1)}^{\min (t, d-1)} \Gamma\left(m+\frac{1}{2}\right) \alpha_{2 m}\left(f_{\mathcal{A}, \lambda} \cdot u_{0, t-m}\right)\right]+O\left(\frac{\left|w_{0}\right|^{-N}}{N^{d+3 / 2}}\right) \\
=\operatorname{Re}\left[w_{0}^{-N} \sum_{t=0}^{d-2} \frac{-4 i}{N^{t+2}} \sum_{m=0}^{t} \Gamma\left(m+\frac{1}{2}\right) \alpha_{2 m}\left(f_{\mathcal{A}, \lambda} \cdot u_{0, t-m}\right)\right]+O\left(\frac{\left|w_{0}\right|^{-N}}{N^{d+1}}\right)
\end{array}
$$

for implied constants depending only on $\lambda^{+}$and $d$. (In going from the previous line to (5.20) we used that $\left|\alpha_{2 m}\left(f_{\mathcal{A}, \lambda} \cdot u_{0, j}\right)\right|$ has a bound depending only on $\lambda^{+}$and $d$, by Proposition 4.4, when $m, j \leqslant d-1$.) Recall that $\mathcal{A}_{1}(N,-\lambda N)=\mathcal{A}_{3}(N,-\lambda N)+O\left(e^{0.05 N}\right)$ by (5.8) and (5.14). Hence, with

$$
a_{t}(\lambda):=-4 i \sum_{m=0}^{t} \Gamma\left(m+\frac{1}{2}\right) \alpha_{2 m}\left(f_{\mathcal{A}, \lambda} \cdot u_{0, t-m}\right),
$$

we obtain (1.17) in the statement of the theorem.

The first coefficient is

$$
a_{0}(\lambda)=-4 i \Gamma(1 / 2) \alpha_{0}\left(f_{\mathcal{A}, \lambda} \cdot u_{0,0}\right)=-4 i \sqrt{\pi} \alpha_{0}\left(f_{\mathcal{A}, \lambda}\right)=-2 i \sqrt{\pi} p_{0}^{1 / 2} f_{\mathcal{A}, \lambda}\left(z_{0}\right),
$$

using (4.3). The terms $p_{0}$ and $q_{0}$ are defined in (4.4) so that, using (4.18) and (5.5),

$$
p_{0}=p^{\prime \prime}\left(z_{0}\right) / 2=\frac{-\pi i e^{2 \pi i z_{0}}}{z_{0} w_{0}}, \quad q_{0}^{2}=q\left(z_{0}\right)^{2}=\frac{i z_{0}}{w_{0}} .
$$

Taking square roots (and numerically checking whether the sign should be + or - ),

$$
p_{0}^{1 / 2}=-\frac{\sqrt{\pi} e^{-\pi i / 4} e^{\pi i z_{0}}}{z_{0}^{1 / 2} w_{0}^{1 / 2}}, \quad q_{0}=-\frac{e^{\pi i / 4} z_{0}^{1 / 2}}{w_{0}^{1 / 2}} .
$$

By (5.9) and (4.4), we have the power series

$$
\begin{aligned}
f_{\mathcal{A}, \lambda}(z)= & f_{0}+f_{1}\left(z-z_{0}\right)+f_{2}\left(z-z_{0}\right)^{2}+\cdots \\
= & e^{-2 \pi i \lambda z_{0}}\left(q_{0}+q_{1}\left(z-z_{0}\right)+q_{2}\left(z-z_{0}\right)^{2}+\cdots\right) \\
& \quad \times\left(1-2 \pi i \lambda\left(z-z_{0}\right)-2 \pi^{2} \lambda^{2}\left(z-z_{0}\right)^{2}+\cdots\right) .
\end{aligned}
$$

This shows that

$$
f_{\mathcal{A}, \lambda}\left(z_{0}\right)=f_{0}=e^{-2 \pi i \lambda z_{0}} q_{0} .
$$

Assembling (5.22), (5.24) and (5.26) proves that $a_{0}(\lambda)=2 z_{0} e^{-\pi i z_{0}(1+2 \lambda)}$. This completes the proof of Theorem 3.1 .

Proposition 5.2. We have

$$
a_{1}(\lambda)=-\frac{w_{0}}{\pi i e^{\pi i z_{0}(3+2 \lambda)}}\left(\frac{\left(2 \pi i z_{0}\right)^{2}}{12}\left(6 \lambda^{2}+6 \lambda+1\right)-2 \pi i z_{0}(2 \lambda+1)+1\right) .
$$

Proof. Formula (5.21) implies

$$
\begin{aligned}
a_{1}(\lambda) & =-4 i\left(\Gamma(1 / 2) \alpha_{0}\left(f_{\mathcal{A}, \lambda} \cdot u_{0,1}\right)+\Gamma(3 / 2) \alpha_{2}\left(f_{\mathcal{A}, \lambda} \cdot u_{0,0}\right)\right) \\
& =-4 i \sqrt{\pi}\left(\alpha_{0}\left(f_{\mathcal{A}, \lambda}\right) \cdot u_{0,1}\left(z_{0}\right)+\alpha_{2}\left(f_{\mathcal{A}, \lambda}\right) / 2\right) .
\end{aligned}
$$


As in [O'S16a, Prop. 5.10], $u_{0,1}\left(z_{0}\right)=\pi i z_{0}\left(-1 / 2+1 / w_{0}\right) / 6$. Also (4.7) implies

$$
\alpha_{2}\left(f_{\mathcal{A}, \lambda}\right)=\frac{1}{2 p_{0}^{1 / 2}} \frac{f_{0}}{p_{0}}\left(\frac{f_{2}}{f_{0}}-\frac{3}{2} \frac{p_{1}}{p_{0}} \frac{f_{1}}{f_{0}}-\frac{3}{2} \frac{p_{2}}{p_{0}}+\frac{15}{8} \frac{p_{1}^{2}}{p_{0}^{2}}\right) .
$$

From (4.17), (4.18) and their generalizations we have

$$
\frac{p_{1}}{p_{0}}=-\frac{1}{z_{0}}+\frac{2 \pi i}{3 w_{0}}, \quad \frac{p_{2}}{p_{0}}=\frac{\pi^{2}}{3 w_{0}}+\frac{1}{z_{0}^{2}}-\frac{2 \pi i}{3 z_{0} w_{0}}-\frac{2 \pi^{2}}{3 w_{0}^{2}} .
$$

Taking derivatives of $q^{2}(z)=i z /\left(1-e^{2 \pi i z}\right)$ and evaluating at $z=z_{0}$ shows that

$$
\frac{q_{1}}{q_{0}}=-\pi i+\frac{1}{2 z_{0}}+\frac{\pi i}{w_{0}}, \quad \frac{q_{2}}{q_{0}}=-\frac{\pi^{2}}{2}-\frac{\pi i}{2 z_{0}}+\frac{2 \pi^{2}}{w_{0}}-\frac{1}{8 z_{0}^{2}}+\frac{\pi i}{2 z_{0} w_{0}}-\frac{3 \pi^{2}}{2 w_{0}^{2}} .
$$

Then by (5.25),

$$
\frac{f_{1}}{f_{0}}=\frac{q_{1}}{q_{0}}-2 \pi i \lambda, \quad \frac{f_{2}}{f_{0}}=\frac{q_{2}}{q_{0}}-2 \pi i \lambda \frac{q_{1}}{q_{0}}-2 \pi^{2} \lambda^{2} .
$$

Putting this all together with the quantities $a_{0}\left(f_{\mathcal{A}, \lambda}\right), p_{0}^{1 / 2}, p_{0}$ and $f_{0}$ evaluated in $(\underline{5.22)}-(\underline{5.26})$, and simplifying with (1.12), finishes the proof.

Table 4 gives examples of the accuracy of Theorem 3.1 , showing the approximation to $\mathcal{A}_{1}(N,-\lambda N)$ on the right side of (3.9) for $N=1200$ and different values of $\lambda$ and $m$. The last column computes $\mathcal{A}_{1}(N,-\lambda N)$ directly from (3.7).

\begin{tabular}{cc|rrrr|r}
$N$ & $\lambda$ & \multicolumn{1}{c}{$m=1$} & \multicolumn{1}{c}{$m=2$} & \multicolumn{1}{c}{$m=3$} & \multicolumn{1}{c}{$m=5$} & \multicolumn{1}{c}{$\mathcal{A}_{1}(N,-\lambda N)$} \\
\hline 1200 & $1 / 3$ & $-1.60733 \times 10^{30}$ & $-1.60827 \times 10^{30}$ & $-1.60783 \times 10^{30}$ & $-1.60784 \times 10^{30}$ & $-1.60784 \times 10^{30}$ \\
1200 & 1 & $1.89943 \times 10^{30}$ & $1.71839 \times 10^{30}$ & $1.72504 \times 10^{30}$ & $1.72506 \times 10^{30}$ & $1.72507 \times 10^{30}$ \\
1200 & 2 & $-1.99478 \times 10^{31}$ & $-1.95514 \times 10^{31}$ & $-1.94125 \times 10^{31}$ & $-1.94292 \times 10^{31}$ & $-1.94291 \times 10^{31}$
\end{tabular}

Table 4: The approximations of Theorem 3.1 to $\mathcal{A}_{1}(N,-\lambda N)$.

\section{Proof of Theorem 3.2}

We prove Theorem 3.2 in this section; it will follow directly from Propositions 6.1, 6.5, 6.7 and 6.10, For $N \geqslant 300$, the indexing set $\mathscr{F}_{N}-\left(\mathscr{F}_{100} \cup \mathcal{A}(N)\right)$ in (3.10) may be partitioned into four pieces:

$$
\mathcal{B}(101, N) \cup \mathcal{C}(N) \cup \mathcal{D}(N) \cup \mathcal{E}(N)
$$

where

$$
\begin{aligned}
& \mathcal{C}(N):=\{h / k: N / 2<k \leqslant N, k \text { odd }, h=2 \text { or } h=k-2\}, \\
& \mathcal{D}(N):=\{h / k: N / 2<k \leqslant N, k \text { odd }, h=(k-1) / 2 \text { or } h=(k+1) / 2\}, \\
& \mathcal{E}(N):=\{h / k: N / 3<k \leqslant N / 2, h=1 \text { or } h=k-1\}
\end{aligned}
$$

and $\mathcal{B}(101, N)$ is what is left. We see next that the sum of $Q_{h k(-\lambda N)}(N)$ for $h / k \in \mathcal{B}(101, N)$ is small enough that we may bound the absolute value of each term. Doing this with the sums over $\mathcal{C}(N), \mathcal{D}(N)$ and $\mathcal{E}(N)$ produces bounds that are larger than the main term of Theorem 3.1 and so we must use saddle-point methods for these. 
6.1 Bounds for $h / k \in \mathcal{B}(101, N)$

Proposition 6.1. Let $\lambda^{+}$be a positive real number. Suppose $N \in \mathbb{Z}_{\geqslant 101}$ and $\lambda N \in \mathbb{Z}$ for $\lambda$ satisfying $|\lambda| \leqslant \lambda^{+}$. For an implied constant depending only on $\lambda^{+}$,

$$
\sum_{h / k \in \mathcal{B}(101, N)} Q_{h k(-\lambda N)}(N)=O\left(e^{0.055 N}\right) .
$$

Proof. For $101 \leqslant k \leqslant N$ and $s:=\lfloor N / k\rfloor$ we have from [O'S16b, Prop. 3.4] that

$$
\left|Q_{h k \sigma}(N)\right| \leqslant \frac{9}{k^{3}} \exp \left(N \frac{2+\log \left(\xi / 2+\xi^{\prime} k / 8\right)}{k}+\frac{|\sigma|}{N}\right)\left|\prod_{N-s k}^{-1}(h / k)\right|
$$

for $\xi \approx 1.00038$ and $\xi^{\prime} \approx 1.01041$. Then (6.5) is used to prove, for an implied constant depending only on $\sigma$, that $\sum_{h / k \in \mathcal{B}(101, N)} Q_{h k \sigma}(N)=O\left(e^{0.055 N}\right)$. This is [O'S16b, Thm. 3.5]. When $\sigma=-\lambda N$, the only $\lambda$ dependence in (6.5) is a factor $\exp (|\lambda|)$.

\subsection{Bounds for $h / k \in \mathcal{C}(N)$}

This section may be read alongside Sections 5 and 6 of [O'S16b]. Set

$$
\mathcal{C}_{1}(N, \sigma):=\sum_{h / k \in \mathcal{C}(N)} Q_{h k \sigma}(N)=2 \operatorname{Re} \sum_{\frac{N}{2}<k \leqslant N, k \text { odd }} Q_{2 k \sigma}(N)
$$

where the equality in (6.6) uses (2.8) and requires $\sigma \in \mathbb{Z}$. We next wish to show

$$
\mathcal{C}_{1}(N,-\lambda N)=O\left(e^{W N}\right)
$$

for a $W<U \approx 0.068$. In fact, the asymptotic expansion of $\mathcal{C}_{1}(N, \sigma)$ is found in [O'S16b, Thm. 1.5] and implies (6.7), but with an implied constant depending on $\sigma$ and hence on $N$ when $\sigma$ takes the form $-\lambda N$. It is straightforward to rework the proof slightly (as we did for $\mathcal{A}_{1}(N,-\lambda N)$ in Section 5) and prove (6.7) with an implied constant depending only on $\lambda$. We give the details of this next.

The sum (6.6) corresponds to $2 N / k \in[2,4)$ and our treatment requires breaking this into two parts: $\mathcal{C}_{2}(N, \sigma)$ for $2 N / k \in[2,3)$ and $\mathcal{C}_{2}^{*}(N, \sigma)$ with $2 N / k \in[3,4)$.

We establish an intermediate result for $\mathcal{C}_{2}(N,-\lambda N)$ first, as follows. Recall $p(z)$ from (5.4) and $g_{\ell}(z)$ from (5.1). Define

$$
\begin{aligned}
q_{\mathcal{C}}(z) & :=\left(\frac{z}{2 \sin (\pi z)}\right)^{1 / 2} \exp (-\pi i z / 2), \\
f_{\mathcal{C}, \lambda}(z) & :=q_{\mathcal{C}}(z) \exp (-2 \pi i \lambda z), \\
v_{\mathcal{C}}(z ; N, \sigma) & :=\frac{2 \pi i \sigma z}{N}+\sum_{\ell=1}^{L-1} \frac{g_{\ell}(z)}{N^{2 \ell-1}} \quad(L=\lfloor 0.006 \pi e \cdot N / 2\rfloor) .
\end{aligned}
$$

Proposition 6.2. Let $\lambda^{+}$be a positive real number. Suppose $N \in \mathbb{Z}_{\geqslant 1}$ and $\lambda N \in \mathbb{Z}$ for $\lambda$ satisfying $|\lambda| \leqslant \lambda^{+}$. For an implied constant depending only on $\lambda^{+}$,

$$
\mathcal{C}_{2}(N,-\lambda N)=\frac{1}{2 N^{3 / 2}} \operatorname{Re} \int_{2.01}^{2.49} \exp (-N \cdot p(z)) f_{\mathcal{C}, \lambda}(z) \exp \left(v_{\mathcal{C}}(z ; N, 0)\right) d z+O\left(e^{0.05 N / 2}\right) .
$$

Proof. The identity

$$
\begin{aligned}
\mathcal{C}_{2}(N, \sigma)=\operatorname{Re} \sum_{k \text { odd, } 2 N / k \in[2,3)} \frac{-2}{k^{2}} \exp & \left(N\left[\frac{\pi i}{2}\left(-\frac{2 N}{k}+5-2 \frac{k}{2 N}\right)\right]\right) \\
& \times \exp \left(\frac{-\pi i}{2} \frac{2 N}{k}\right) \exp \left(\frac{1}{N}\left[2 \pi i \sigma \frac{2 N}{k}\right]\right) \prod_{N-k}^{-1}(2 / k) .
\end{aligned}
$$


is [O'S16b, eq. (5.3)]. Set $\hat{z}=\hat{z}(N, k):=2 N / k$ and define

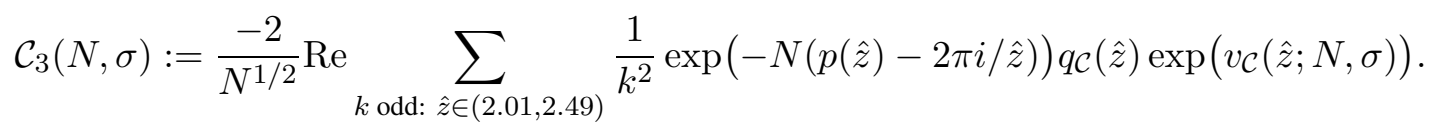

The sine product $\prod_{N-k}^{-1}(2 / k)$ in (6.12) may be estimated precisely using Euler-Maclaurin summation as in ['S16b, Thm. 5.1]. The result is that $\mathcal{C}_{2}(N, \sigma)$ and $\mathcal{C}_{3}(N, \sigma)$ differ by at most $O\left(e^{0.05 N / 2}\right)$ for an absolute implied constant. For $\sigma=-\lambda N$ we easily obtain

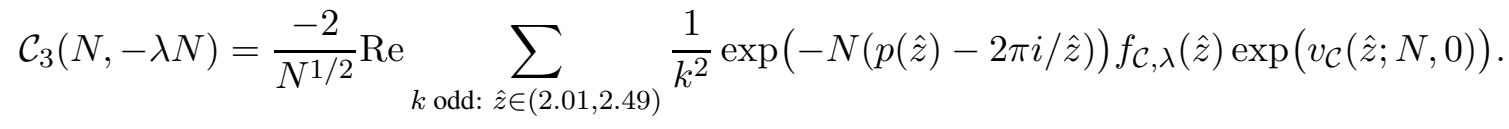

It is shown in [O'S16b, Thm. 5.4] that $q_{\mathcal{C}}(z)$ and $v_{\mathcal{C}}(z ; N, 0)$ are holomorphic and absolutely bounded on a domain containing the box $\mathbb{B}_{2}$, defined in (5.11). Hence,

$$
f_{\mathcal{C}, \lambda}(z) \exp \left(v_{\mathcal{C}}(z ; N, 0)\right) \ll 1 \quad \text { for } \quad z \in \mathbb{B}_{2}
$$

with an implied constant depending only on $\lambda^{+}$. Using this bound, the proofs of Propositions 5.6 and 5.7 in [O'S16b] go through. This gives the desired result, expressing $\mathcal{C}_{2}(N,-\lambda N)$ as the integral in (6.11).

The second component, $\mathcal{C}_{2}^{*}(N, \sigma)$, is treated in a similar way as follows. Define

$$
\begin{aligned}
p_{1}(z) & :=\frac{1}{2 \pi i z}\left[-\operatorname{Li}_{2}\left(e^{2 \pi i z}\right)+\operatorname{Li}_{2}(1)+4 \pi^{2}\right], \\
q_{\mathcal{C}}^{*}(z) & :=e^{-3 \pi i / 4} \sqrt{z}, \\
f_{\mathcal{C}, \lambda}^{*}(z) & :=q_{\mathcal{C}}^{*}(z) \exp (-2 \pi i \lambda z), \\
v_{\mathcal{C}}^{*}(z ; N, \sigma) & :=\frac{\pi i(16 \sigma+1) z}{8(N+1 / 2)}+\sum_{\ell=1}^{L-1} \frac{g_{\ell}(z)}{(2(N+1 / 2))^{2 \ell-1}}-\sum_{\ell=1}^{L^{*}-1} \frac{g_{\ell}(z)}{(N+1 / 2)^{2 \ell-1}}
\end{aligned}
$$

for $L=\lfloor 0.006 \pi e \cdot 2 N / 3\rfloor$ and $L^{*}=\lfloor 0.006 \pi e \cdot N / 3\rfloor$.

Proposition 6.3. Let $\lambda^{+}$be a positive real number. Suppose $N \in \mathbb{Z}_{\geqslant 1}$ and $\lambda N \in \mathbb{Z}$ for $\lambda$ satisfying $|\lambda| \leqslant \lambda^{+}$. For an implied constant depending only on $\lambda^{+}$,

$$
\begin{array}{r}
\mathcal{C}_{2}^{*}(N,-\lambda N)=\frac{-1}{4(N+1 / 2)^{3 / 2}} \operatorname{Im} \int_{3.01}^{3.49} \exp \left(-(N+1 / 2) p_{1}(z)\right) f_{\mathcal{C}, \lambda}^{*}(z) \exp \left(v_{\mathcal{C}}^{*}(z ; N, \lambda / 2)\right) d z \\
+O\left(e^{0.05 N / 3}\right)
\end{array}
$$

Proof. This time we set $\hat{z}:=2(N+1 / 2) / k$ and define

$$
\begin{aligned}
& \mathcal{C}_{3}^{*}(N, \sigma):=\frac{1}{(N+1 / 2)^{1 / 2}} \operatorname{Re} \sum_{k \text { odd }: \hat{z} \in(3.01,3.49)} \frac{(-1)^{(k+1) / 2}}{k^{2}} \\
& \times \exp \left(-(N+1 / 2)\left(p_{1}(\hat{z})-3 \pi i / \hat{z}\right)\right) q_{\mathcal{C}}^{*}(\hat{z}) \exp \left(v_{\mathcal{C}}^{*}(\hat{z} ; N, \sigma)\right) .
\end{aligned}
$$

It is shown in [O'S16b, eq. (6.14)] that $\mathcal{C}_{2}^{*}(N, \sigma)$ and $\mathcal{C}_{3}^{*}(N, \sigma)$ differ by at most $O\left(e^{0.05 N / 3}\right)$ for an absolute implied constant. We may replace $q_{\mathcal{C}}^{*}(z) \exp \left(v_{\mathcal{C}}^{*}(z ; N, \sigma)\right)$ in (6.17) by $f_{\mathcal{C}, \lambda}^{*}(z) \exp \left(v_{\mathcal{C}}^{*}(z ; N, \lambda / 2)\right)$ since $\sigma=-\lambda N$. Put

$$
g_{\mathcal{C}, \ell}(z):=g_{\ell}(z)\left(2^{-(2 \ell-1)}-1\right)
$$

and the next result is [O'S16b, Corollary 6.4]. 
Lemma 6.4. For $\delta=0.007$ and all $z \in \mathbb{C}$ with $3+\delta \leqslant \operatorname{Re}(z) \leqslant 3.5-\delta$, we have

$$
v_{\mathcal{C}}^{*}(z ; N, \sigma)=\frac{\pi i(16 \sigma+1) z}{8(N+1 / 2)}+\sum_{\ell=1}^{d-1} \frac{g_{\mathcal{C}, \ell}(z)}{(N+1 / 2)^{2 \ell-1}}+O\left(\frac{1}{N^{2 d-1}}\right)
$$

where $2 \leqslant d \leqslant L^{*}=\lfloor 0.006 \pi e \cdot N / 3\rfloor$ and the implied constant depends only on $d$.

With the above lemma we may show that $f_{\mathcal{C}, \lambda}^{*}(z) \exp \left(v_{\mathcal{C}}^{*}(z ; N, \lambda / 2)\right)$ is holomorphic on a domain containing $\mathbb{B}_{3}$, and that

$$
f_{\mathcal{C}, \lambda}^{*}(z) \exp \left(v_{\mathcal{C}}^{*}(z ; N, \lambda / 2)\right) \ll 1 \quad \text { for } \quad z \in \mathbb{B}_{3}
$$

with an implied constant depending only on $\lambda^{+}$. The proof in Sections 6.2 and 6.3 of [O'S16b] now goes through and we obtain our integral representation (6.16).

Proposition 6.5. Let $\lambda^{+}$be a positive real number. Suppose $N \in \mathbb{Z}_{\geqslant 1}$ and $\lambda N \in \mathbb{Z}$ for $\lambda$ satisfying $|\lambda| \leqslant \lambda^{+}$. For an implied constant depending only on $\lambda^{+}$,

$$
\mathcal{C}_{1}(N,-\lambda N)=O\left(e^{0.036 N}\right) .
$$

Proof. We bound the integral representation (6.11) of $\mathcal{C}_{2}(N,-\lambda N)$ by first moving the path of integration to one going through a saddle-point of $p(z)$. By Theorem 4.6, the unique solution to $p^{\prime}(z)=0$ for $1.5<$ $\operatorname{Re}(z)<2.5$ is

$$
z_{1}:=2+\frac{\log (1-w(0,-2))}{2 \pi i} \approx 2.20541+0.345648 i
$$

Let $c=1+i \operatorname{Im}\left(z_{1}\right) / \operatorname{Re}\left(z_{1}\right)$ and make $\mathcal{Q}$ the polygonal path between the points 2.01, 2.01c, 2.49c and 2.49, as shown in Figure 1, passing through $z_{1}$. It is proved in [O'S16b, Thm. 5.8] that

$$
\operatorname{Re}\left(p(z)-p\left(z_{1}\right)\right)>0 \text { for all } z \in \mathcal{Q}, z \neq z_{1} .
$$

Therefore, recalling 6.14),

$$
\mathcal{C}_{2}(N,-\lambda N) \ll e^{U_{\mathcal{C}} N} \quad \text { for } \quad U_{\mathcal{C}}:=-\operatorname{Re}\left(p\left(z_{1}\right)\right)=-\log |w(0,-2)| \approx 0.0256706 .
$$

Bounding the integral representation (6.16) of $\mathcal{C}_{2}^{*}(N,-\lambda N)$ is achieved similarly. By Theorem 4.6, the unique solution to $p_{1}^{\prime}(z)=0$ for $2.5<\operatorname{Re}(z)<3.5$ is

$$
z_{3}:=3+\frac{\log (1-w(1,-3))}{2 \pi i} \approx 3.08382-0.0833451 i .
$$

Let $c=1+i \operatorname{Im}\left(z_{3}\right) / \operatorname{Re}\left(z_{3}\right)$ and make $\mathcal{S}$ the polygonal path between the points 3.01, 3.01c, 3.49c and 3.49, as shown in Figure 1, passing through $z_{3}$. As seen in [O'S16b, Sect. 6.3],

$$
\operatorname{Re}\left(p_{1}(z)-p_{1}\left(z_{3}\right)\right)>0 \text { for all } z \in \mathcal{S}, z \neq z_{3} .
$$

Therefore, recalling 6.19),

$$
\mathcal{C}_{2}^{*}(N,-\lambda N) \ll e^{U_{\mathcal{C}}^{*} N} \quad \text { for } \quad U_{\mathcal{C}}^{*}:=-\operatorname{Re}\left(p\left(z_{3}\right)\right)=-\log |w(1,-3)| \approx 0.0356795 .
$$

Together, 6.22) and (6.23) prove 6.20). 
6.3 Bounds for $h / k \in \mathcal{D}(N)$

This section may be read alongside Section 7 of [O'S16b]. Write

$$
\mathcal{D}_{1}(N, \sigma):=\sum_{h / k \in \mathcal{D}(N)} Q_{h k \sigma}(N)=2 \operatorname{Re} \sum_{\frac{N}{2}<k \leqslant N, k \text { odd }} Q_{\left(\frac{k-1}{2}\right) k \sigma}(N) .
$$

Recall $g_{\ell}(z)$ from (5.1) and define

$$
\begin{aligned}
q_{\mathcal{D}}(z) & :=\left(\frac{z}{2 \sin (\pi(z-1) / 2)}\right)^{1 / 2} \exp \left(-\frac{\pi i}{4}(z+3)\right), \\
q_{\mathcal{D}}^{*}(z) & :=2 \sin (\pi(z-1) / 2)\left(\frac{z}{2 \sin (\pi(z-1) / 2)}\right)^{1 / 2} \exp \left(\frac{\pi i}{4}(z-1)\right), \\
g_{\ell}^{*}(z) & :=-\frac{B_{2 \ell}}{(2 \ell) !}(\pi z / 2)^{2 \ell-1} \cot ^{(2 \ell-2)}(\pi(z-1) / 2), \\
v_{\mathcal{D}}(z ; N, \sigma) & :=\frac{\pi i \sigma z}{N}+\sum_{\ell=1}^{L-1} \frac{g_{\ell}(z)-g_{\ell}^{*}(z)}{N^{2 \ell-1}}+\sum_{\ell=1}^{L^{*}-1} \frac{2 g_{\ell}^{*}(z)-g_{\ell}(z)}{(N / 2)^{2 \ell-1}}
\end{aligned}
$$

for $L:=\lfloor 0.006 \pi e \cdot N\rfloor$ and $L^{*}:=\lfloor 0.006 \pi e \cdot N / 2\rfloor$. Also set

$$
f_{\mathcal{D}, \lambda}(z):=q_{\mathcal{D}}(z) \exp (-\pi i \lambda z), \quad f_{\mathcal{D}, \lambda}^{*}(z):=i q_{\mathcal{D}}^{*}(z) \exp (-\pi i \lambda z) .
$$

Proposition 6.6. Let $\lambda^{+}$be a positive real number. Suppose $N \in \mathbb{Z}_{\geqslant 1}$ and $\lambda N \in \mathbb{Z}$ for $\lambda$ satisfying $|\lambda| \leqslant \lambda^{+}$. Then

$$
\mathcal{D}_{1}(N,-\lambda N)=-\operatorname{Re} \int_{1.01}^{1.49} \frac{\exp (-N \cdot p(z) / 2)}{N^{3 / 2}} f_{\mathcal{D}, \lambda}(z) \exp \left(v_{\mathcal{D}}(z ; N, 0)\right) d z+O\left(e^{0.05 N / 2}\right)
$$

for $N$ odd. Also

$$
\mathcal{D}_{1}(N,-\lambda N)=-\operatorname{Re} \int_{1.01}^{1.49} \frac{\exp (-(N+1) \cdot p(z) / 2)}{(N+1)^{3 / 2}} f_{\mathcal{D}, \lambda}^{*}(z) \exp \left(v_{\mathcal{D}}(z ; N+1, \lambda)\right) d z+O\left(e^{0.05 N / 2}\right)
$$

for $N$ even. The implied constants in (6.26) and (6.27) depend only on $\lambda^{+}$,

Proof. For $N$ odd, the summands in (6.24) satisfy the identity

$$
\begin{aligned}
Q_{\left(\frac{k-1}{2}\right) k \sigma}(N)=\frac{1}{k^{2}} \exp ( & \left.N\left[\frac{\pi i}{4}\left(\frac{N}{k}+1+\frac{2 k}{N}\right)\right]\right) \\
& \times \exp \left(\frac{\pi i}{4}\left(\frac{N}{k}+3\right)\right) \exp \left(\frac{1}{N}\left[-\pi i \sigma \frac{N}{k}\right]\right) \prod_{N-k}^{-1}((k-1) / 2 k)
\end{aligned}
$$

which is [O'S16b, Eq. (7.2)]. Next put $\hat{z}:=N / k$ and

$$
\mathcal{D}_{2}(N, \sigma):=\frac{-2}{N^{1 / 2}} \operatorname{Re} \sum_{k \text { odd }:} \sum_{z \in(1.01,1.49)} \frac{1}{k^{2}} \exp (-N \cdot p(\hat{z}) / 2) q_{\mathcal{D}}(\hat{z}) \exp \left(v_{\mathcal{D}}(\hat{z} ; N, \sigma)\right)
$$

for $N$ odd. It is shown in [O'S16b, Eq. (7.30)] that $\mathcal{D}_{1}(N, \sigma)$ and $\mathcal{D}_{2}(N, \sigma)$ differ by at most $O\left(e^{0.05 N / 2}\right)$ for an absolute implied constant. With $\sigma=-\lambda N$ we substitute

$$
f_{\mathcal{D}, \lambda}(z) \exp \left(v_{\mathcal{D}}(z ; N, 0)\right)=q_{\mathcal{D}}(z) \exp \left(v_{\mathcal{D}}(z ; N, \sigma) .\right.
$$

in [6.29). It follows from [O'S16b, Thm. 7.5] that $f_{\mathcal{D}, \lambda}(z) \exp \left(v_{\mathcal{D}}(z ; N, 0)\right)$ is holomorphic on a domain containing $\mathbb{B}_{1}$ and

$$
f_{\mathcal{D}, \lambda}(z) \exp \left(v_{\mathcal{D}}(z ; N, 0)\right) \ll 1 \quad \text { for } \quad z \in \mathbb{B}_{1}
$$


with an implied constant depending only on $\lambda^{+}$. The proof in Section 7.2 of [O'S16b] now goes through and we obtain our integral representation (6.26). The case where $N$ is even is very similar - see Section 7.3 of [O'S16b] - using

$$
\begin{aligned}
\mathcal{D}_{2}(N, \sigma):=\frac{-2}{(N+1)^{1 / 2}} \operatorname{Re} \sum_{k \text { odd }: \hat{z} \in(1.01,1.49)} \frac{(-1)^{(k+1) / 2}}{k^{2}} \\
\quad \times \exp \left(-(N+1)\left[\frac{p(\hat{z})}{2}+\frac{\pi i}{2 \hat{z}}\right]\right) q_{\mathcal{D}}^{*}(\hat{z}) \exp \left(v_{\mathcal{D}}(\hat{z} ; N+1, \sigma)\right) \quad(N \text { even }) .
\end{aligned}
$$

Proposition 6.7. Let $\lambda^{+}$be a positive real number. Suppose $N \in \mathbb{Z}_{\geqslant 1}$ and $\lambda N \in \mathbb{Z}$ for $\lambda$ satisfying $|\lambda| \leqslant \lambda^{+}$. Then for an implied constant depending only on $\lambda^{+}$,

$$
\mathcal{D}_{1}(N,-\lambda N)=O\left(e^{0.035 N}\right) .
$$

Proof. Change the path of integration in (6.26) and (6.27) to $\mathcal{P}$, as described in Section 5 , We saw there that

$$
\operatorname{Re}\left(p(z)-p\left(z_{0}\right)\right)>0 \text { for all } z \in \mathcal{P}, z \neq z_{0} .
$$

It now follows from (6.30) (and the analogous bound for $N$ even) that

$$
\mathcal{D}_{1}(N,-\lambda N) \ll e^{U N / 2} \quad \text { for } \quad U:=-\operatorname{Re}\left(p\left(z_{0}\right)\right)=-\log |w(0,-1)| \approx 0.0680762
$$

(as in (1.15), 5.16) ) and we have completed the proof.

\subsection{Bounds for $h / k \in \mathcal{E}(N)$}

This section may be read alongside Section 8 of [O'S16b]. Put

$$
\mathcal{E}_{1}(N, \sigma):=\sum_{h / k \in \mathcal{E}(N)} Q_{h k \sigma}(N)=2 \operatorname{Re} \sum_{\frac{N}{3}<k \leqslant \frac{N}{2}} Q_{1 k \sigma}(N) .
$$

To express (6.34) as an integral we need the next definitions. First set

$$
\tilde{g}_{\ell}(z):=\frac{B_{2 \ell}}{(2 \ell) !}(\pi z)^{2 \ell-1}\left\{\pi z \cot ^{(2 \ell-1)}(\pi z)+(2 \ell-1) \cot ^{(2 \ell-2)}(\pi z)\right\} .
$$

Then we define the function $\phi_{\sigma, m}(z)$ in the following way:

$$
\begin{aligned}
\phi_{\sigma, 0}(z) & :=\frac{1}{4 \pi^{2}}\left[\operatorname{Li}_{2}(1)-\operatorname{Li}_{2}\left(e^{2 \pi i z}\right)+6 \pi^{2}-2 \pi i z \log \left(1-e^{2 \pi i z}\right)\right] \\
\phi_{\sigma, 1}(z) & :=\frac{z^{2} \cot (\pi z)}{4 i}+\frac{z^{2}}{4}-\frac{5 z}{4 \pi i} \\
\phi_{\sigma, 2}(z) & :=-\sigma z^{2}+\frac{z \tilde{g}_{1}(z)}{2 \pi i}
\end{aligned}
$$

and for $m \in \mathbb{Z}_{\geqslant 3}$

$$
\phi_{\sigma, m}(z):= \begin{cases}z \tilde{g}_{m / 2}(z) /(2 \pi i) & \text { if } m \text { is even } \\ 0 & \text { if } m \text { is odd. }\end{cases}
$$

Clearly, only $\phi_{\sigma, 2}(z)$ depends on $\sigma$. With $q_{\mathcal{C}}(z)$ given in (6.8), put

$$
q_{\mathcal{E}}(z ; N, \sigma):=q_{\mathcal{C}}(z) \times \sum_{\ell=0}^{2 L-1} \frac{\phi_{\sigma, \ell}(z)}{N^{\ell}}
$$

for $L:=\lfloor 0.006 \pi e \cdot N / 2\rfloor$ and finally define

$$
f_{\mathcal{E}, \lambda}(z ; N):=q_{\mathcal{E}}(z ; N,-\lambda N) \exp (-2 \pi i \lambda z) .
$$


Proposition 6.8. Let $\lambda^{+}$be a positive real number. Suppose $N \in \mathbb{Z}_{\geqslant 1}$ and $\lambda N \in \mathbb{Z}$ for $\lambda$ satisfying $|\lambda| \leqslant \lambda^{+}$. Then for an implied constant depending only on $\lambda^{+}$,

$$
\mathcal{E}_{1}(N,-\lambda N)=\frac{1}{N^{3 / 2}} \operatorname{Re} \int_{2.01}^{2.49} \exp (-N \cdot p(z)) f_{\mathcal{E}, \lambda}(z ; N) \exp \left(v_{\mathcal{C}}(z ; N, 0)\right) d z+O\left(N e^{0.05 N / 2}\right)
$$

Proof. For $N / 3<k \leqslant N / 2$ and $\sigma \in \mathbb{Z}$, we know by [O'S16b, Eqs. (8.3), (8.4)] that

$$
\begin{aligned}
Q_{1 k \sigma}(N)=\frac{1}{2 k^{2}} \phi(N, k, \sigma) \exp \left(N \left[\frac{-i \pi}{2}\right.\right. & \left.\left.\left(\frac{N}{k}-1+2 \frac{k}{N}\right)\right]\right) \\
& \times \exp \left(\frac{-i \pi}{2} \frac{N}{k}\right) \exp \left(\frac{1}{N}\left[2 i \pi \sigma \frac{N}{k}\right]\right) \prod_{N-2 k}^{-1}(1 / k)
\end{aligned}
$$

where

$$
\phi(N, k, \sigma):=\frac{1}{4 k^{2}}\left(N^{2}+N-4 \sigma\right)+\frac{1}{2 \pi i k} \sum_{1 \leqslant j \leqslant N, k \nmid j} \frac{\pi j}{k} \cot \left(\frac{\pi j}{k}\right) .
$$

Let $\hat{z}=N / k$ again. It is shown in [O'S16b, Sections 8.3, 8.4] that

$$
\phi(N, k, \sigma)=\sum_{\ell=0}^{2 L-1} \frac{\phi_{\sigma, \ell}(\hat{z})}{N^{\ell}}+\frac{\varepsilon_{L}(N-2 k, 1 / k)}{2 \pi i k}
$$

with error term $\varepsilon_{L}$ satisfying

$$
\begin{aligned}
\prod_{N-2 k}^{-1}(1 / k) \frac{\varepsilon_{L}(N-2 k, 1 / k)}{2 \pi i k} & =O\left(N e^{0.05 N / 2}\right), \\
\frac{\varepsilon_{L}(N-2 k, 1 / k)}{2 \pi i k} & =O(N)
\end{aligned}
$$

where $L=\lfloor 0.006 \pi e \cdot N / 2\rfloor$ as in the definition of $q_{\mathcal{E}}$. Recalling $(6.10)$, we next set

$$
\mathcal{E}_{2}(N, \sigma):=\frac{1}{N^{1 / 2}} \operatorname{Re} \sum_{k: \hat{z} \in(2.01,2.49)} \frac{1}{k^{2}} \exp (-N(p(\hat{z})-2 \pi i / \hat{z})) q_{\mathcal{E}}(\hat{z} ; N, \sigma) \exp \left(v_{\mathcal{C}}(\hat{z} ; N, \sigma)\right) .
$$

It is proved in [O'S16b, Prop. 8.5] (using (6.40) and (6.41) that $\mathcal{E}_{2}(N, \sigma)=\mathcal{E}_{1}(N, \sigma)+O\left(N e^{0.05 N / 2}\right)$ for an implied constant depending only on $\sigma$. To adapt this to the case that $\sigma=-\lambda N$, we need the next result.

Lemma 6.9. For $N / 3<k \leqslant N / 2,|\lambda| \leqslant \lambda^{+}$and an implied constant depending only on $\lambda^{+}$

$$
\phi(N, k,-\lambda N)=O(N) .
$$

Proof. Verify that $|\cot (\pi j / k)|<2 k / \pi$ when $k \nmid j$. This implies

$$
\phi(N, k,-\lambda N) \ll 1+\frac{|\lambda| N}{k^{2}}+\frac{1}{k} \sum_{j=1}^{N} \frac{j}{k} k \ll \frac{|\lambda|}{N}+N .
$$

The proof of [O'S16b, Prop. 8.5] with $\sigma=-\lambda N$ and the bound from Lemma 6.9 now shows that

$$
\mathcal{E}_{2}(N,-\lambda N)=\mathcal{E}_{1}(N,-\lambda N)+O\left(N e^{0.05 N / 2}\right)
$$

for an implied constant depending only on $\lambda^{+}$. Replacing $\sigma$ by $-\lambda N$ also lets us rewrite $q_{\mathcal{E}}$ as

$$
q_{\mathcal{E}}(z ; N,-\lambda N)=q_{\mathcal{C}}(z) \times \sum_{\ell=0}^{2 L-1} \frac{\phi_{\lambda, \ell}^{*}(z)}{N^{\ell}}
$$


with

$$
\phi_{\lambda, \ell}^{*}(z):= \begin{cases}\phi_{0, \ell}(z) & \text { if } \quad \ell \in \mathbb{Z}_{\geqslant 0}, \ell \neq 1 \\ \phi_{0,1}(z)+\lambda z^{2} & \text { if } \quad \ell=1 .\end{cases}
$$

In this way, $\phi_{\lambda, \ell}^{*}(z)$ is always independent of $N$, and only depends on $\lambda$ when $\ell=1$. We easily obtain

$$
\mathcal{E}_{2}(N,-\lambda N)=\frac{1}{N^{1 / 2}} \operatorname{Re} \sum_{k: \hat{z} \in(2.01,2.49)} \frac{1}{k^{2}} \exp (-N(p(\hat{z})-2 \pi i / \hat{z})) f_{\mathcal{E}, \lambda}(\hat{z} ; N) \exp \left(v_{\mathcal{C}}(\hat{z} ; N, 0)\right) .
$$

With the help of [O'S16b, Lemma 8.6] we see that $f_{\mathcal{E}, \lambda}(z ; N) \exp \left(v_{\mathcal{C}}(z ; N, 0)\right)$ is holomorphic on a domain containing $\mathbb{B}_{2}$ and that

$$
f_{\mathcal{E}, \lambda}(z ; N) \exp \left(v_{\mathcal{C}}(z ; N, 0)\right) \ll 1 \quad \text { for } \quad z \in \mathbb{B}_{2}
$$

with an implied constant depending only on $\lambda^{+}$. The proof in Section 8.4 of [O'S16b] now goes through and we obtain our integral representation (6.37).

Proposition 6.10. Let $\lambda^{+}$be a positive real number. Suppose $N \in \mathbb{Z}_{\geqslant 1}$ and $\lambda N \in \mathbb{Z}$ for $\lambda$ satisfying $|\lambda| \leqslant \lambda^{+}$. Then for an implied constant depending only on $\lambda^{+}$,

$$
\mathcal{E}_{1}(N,-\lambda N)=O\left(e^{0.026 N}\right) .
$$

Proof. As in the first part of the proof of Proposition 6.5, we move the path of integration of (6.37) to $\mathcal{Q}$ passing through the saddle-point $z_{1}$. As we have seen in (6.21) and (6.22),

$$
-\operatorname{Re}(p(z)) \leqslant-\operatorname{Re}\left(p\left(z_{1}\right)\right)=-\log |w(0,-2)| \approx 0.0256706
$$

for $z \in \mathcal{Q}$. Combining this bound with (6.44) completes the proof.

This last proposition completes the proof of Theorem 3.2. This finishes the proof of Theorem 1.2 as well.

\section{Asymptotic expansions for $\mathcal{C}_{1}, \mathcal{D}_{1}$ and $\mathcal{E}_{1}$}

We may continue the analysis of $\mathcal{C}_{1}(N,-\lambda N), \mathcal{D}_{1}(N,-\lambda N)$ and $\mathcal{E}_{1}(N,-\lambda N)$ to obtain their asymptotic expansions. While not necessary to prove Theorem 3.2 , this allows us to numerically verify our work, as we see in Tables 5- 8, and also suggests some further results that we describe in Section 9

Recall the dilogarithm zeros $w_{0}=w(0,-1), w(0,-2)$ and $w(1,-3)$ from Section 4.2, Starting with Proposition 6.2, a similar proof to Theorem 3.1 shows the asymptotic expansion of $\mathcal{C}_{2}(N,-\lambda N)$, the first component of $\mathcal{C}_{1}(N,-\lambda N)$. Details are much the same as [O'S16b, Sect. 5.4]. The functions $u_{0, j}$ and $f_{\mathcal{C}, \lambda}$ are defined in (5.17) and (6.9), respectively.

Theorem 7.1. Let $\lambda^{+}$be a positive real number. Suppose $N \in \mathbb{Z}_{\geqslant 1}$ and $\lambda N \in \mathbb{Z}$ for $\lambda$ satisfying $|\lambda| \leqslant \lambda^{+}$. We have

$$
\mathcal{C}_{2}(N,-\lambda N)=\operatorname{Re}\left[\frac{w(0,-2)^{-N}}{N^{2}}\left(c_{0}(\lambda)+\frac{c_{1}(\lambda)}{N}+\cdots+\frac{c_{m-1}(\lambda)}{N^{m-1}}\right)\right]+O\left(\frac{|w(0,-2)|^{-N}}{N^{m+2}}\right)
$$

for an implied constant depending only on $\lambda^{+}$and $m$. The functions $c_{t}(\lambda)$ are given as follows, where $\alpha$ depends on $p$ with saddle-point $z_{1}$,

$$
c_{t}(\lambda)=\sum_{s=0}^{t} \Gamma\left(s+\frac{1}{2}\right) \alpha_{2 s}\left(f_{\mathcal{C}, \lambda} \cdot u_{0, t-s}\right) .
$$

A comparison of both sides of (7.1) in Theorem 7.1 with some different values of $N, \lambda$ and $m$ is shown in Table 5. The asymptotic expansion of $\mathcal{C}_{2}^{*}(N,-\lambda N)$, the second component of $\mathcal{C}_{1}(N,-\lambda N)$, is given next based on a similar development in [O'S16b, Sect. 6.4]. Recall $f_{\mathcal{C}, \lambda}^{*}$ from (6.15) and $g_{\mathcal{C}, \ell}$ from (6.18). Set $u_{\sigma, 0}^{*}:=1$ and for $j \in \mathbb{Z}_{\geqslant 1}$ put

$$
u_{\sigma, j}^{*}(z):=\sum_{m_{1}+3 m_{2}+5 m_{3}+\cdots=j} \frac{\left(\pi i(16 \sigma+1) z / 8+g_{\mathcal{C}, 1}(z)\right)^{m_{1}}}{m_{1} !} \frac{g_{\mathcal{C}, 2}(z)^{m_{2}}}{m_{2} !} \cdots \frac{g_{\mathcal{C}, j}(z)^{m_{j}}}{m_{j} !} .
$$




\begin{tabular}{cc|ccrr|r}
$N$ & $\lambda$ & $m=1$ & $m=2$ & \multicolumn{1}{c}{$m=3$} & \multicolumn{1}{c}{$m=5$} & \multicolumn{1}{c}{$\mathcal{C}_{2}(N,-\lambda N)$} \\
\hline 1200 & $1 / 3$ & $3.82015 \times 10^{7}$ & $3.94694 \times 10^{7}$ & $3.92935 \times 10^{7}$ & $3.93016 \times 10^{7}$ & $3.93016 \times 10^{7}$ \\
1200 & 1 & $-7.55408 \times 10^{7}$ & $-9.44498 \times 10^{7}$ & $-8.92043 \times 10^{7}$ & $-8.98686 \times 10^{7}$ & $-8.98650 \times 10^{7}$ \\
1200 & 2 & $-4.19152 \times 10^{9}$ & $-3.06226 \times 10^{9}$ & $-3.20956 \times 10^{9}$ & $-3.20879 \times 10^{9}$ & $-3.21242 \times 10^{9}$
\end{tabular}

Table 5: The approximations of Theorem 7.1 to $\mathcal{C}_{2}(N,-\lambda N)$.

Theorem 7.2. Let $\lambda^{+}$be a positive real number. Suppose $N \in \mathbb{Z}_{\geqslant 1}$ and $\lambda N \in \mathbb{Z}$ for $\lambda$ satisfying $|\lambda| \leqslant \lambda^{+}$. We have

$$
\mathcal{C}_{2}^{*}(N,-\lambda N)=\operatorname{Re}\left[\frac{w(1,-3)^{-N}}{N^{2}}\left(c_{0}^{*}(\lambda)+\frac{c_{1}^{*}(\lambda)}{N}+\cdots+\frac{c_{m-1}^{*}(\lambda)}{N^{m-1}}\right)\right]+O\left(\frac{|w(1,-3)|^{-N}}{N^{m+2}}\right)
$$

for an implied constant depending only on $\lambda^{+}$and $m$. The functions $c_{t}^{*}(\lambda)$ are given as follows, where $\alpha$ depends on $p_{1}$ with saddle-point $z_{3}$,

$$
c_{t}^{*}(\lambda)=\frac{w(1,-3)^{-1 / 2}}{2} \sum_{j=0}^{t}(-2)^{j-t}\left(\begin{array}{c}
t+1 \\
j+1
\end{array}\right) \sum_{s=0}^{j} \Gamma\left(s+\frac{1}{2}\right) \alpha_{2 s}\left(i f_{\mathcal{C}, \lambda}^{*} \cdot u_{\lambda / 2, j-s}^{*}\right) .
$$

\begin{tabular}{cc|cccc|c}
$N$ & $\lambda$ & $m=1$ & $m=2$ & $m=3$ & $m=5$ & $\mathcal{C}_{2}^{*}(N,-\lambda N)$ \\
\hline 1200 & $1 / 3$ & $-3.41978 \times 10^{11}$ & $-3.38619 \times 10^{11}$ & $-3.38622 \times 10^{11}$ & $-3.38622 \times 10^{11}$ & $-3.38622 \times 10^{11}$ \\
1200 & 1 & $-5.45974 \times 10^{11}$ & $-5.36354 \times 10^{11}$ & $-5.36405 \times 10^{11}$ & $-5.36404 \times 10^{11}$ & $-5.36404 \times 10^{11}$ \\
1200 & 2 & $-5.14590 \times 10^{11}$ & $-5.00599 \times 10^{11}$ & $-5.00740 \times 10^{11}$ & $-5.00737 \times 10^{11}$ & $-5.00737 \times 10^{11}$
\end{tabular}

Table 6: The approximations of Theorem 7.2 to $\mathcal{C}_{2}^{*}(N,-\lambda N)$.

The detailed expansion of $\mathcal{D}_{1}(N,-\lambda N)$ is derived similarly to Section 7 of [O'S16b]. We need $f_{\mathcal{D}, \lambda}$, $f_{\mathcal{D}, \lambda}^{*}$ from 6.25). Also set

$$
g_{\mathcal{D}, \ell}(z):=g_{\ell}(z)-g_{\ell}^{*}(z)+2^{2 \ell-1}\left(2 g_{\ell}^{*}(z)-g_{\ell}(z)\right)
$$

and

$$
u_{\mathcal{D}, \sigma, j}(z):=\sum_{m_{1}+3 m_{2}+5 m_{3}+\cdots=j} \frac{\left(\pi i \sigma z+g_{\mathcal{D}, 1}(z)\right)^{m_{1}}}{m_{1} !} \frac{g_{\mathcal{D}, 2}(z)^{m_{2}}}{m_{2} !} \cdots \frac{g_{\mathcal{D}, j}(z)^{m_{j}}}{m_{j} !},
$$

with $u_{\mathcal{D}, \sigma, 0}=1$.

Theorem 7.3. Let $\lambda^{+}$be a positive real number. Suppose $N \in \mathbb{Z}_{\geqslant 1}$ and $\lambda N \in \mathbb{Z}$ for $\lambda$ satisfying $|\lambda| \leqslant \lambda^{+}$. With $\bar{N}$ denoting $N \bmod 2$, we have

$$
\mathcal{D}_{1}(N,-\lambda N)=\operatorname{Re}\left[\frac{w_{0}^{-N / 2}}{N^{2}}\left(d_{0}(\lambda, \bar{N})+\frac{d_{1}(\lambda, \bar{N})}{N}+\cdots+\frac{d_{m-1}(\lambda, \bar{N})}{N^{m-1}}\right)\right]+O\left(\frac{\left|w_{0}\right|^{-N / 2}}{N^{m+2}}\right)
$$

for an implied constant depending only on $\lambda^{+}$and $m$. The functions $d_{t}(\lambda, \bar{N})$ are given as follows, where $\alpha$ depends on $p / 2$ with saddle-point $z_{0}$,

$$
\begin{aligned}
& d_{t}(\lambda, \overline{1})=-2 \sum_{s=0}^{t} \Gamma\left(s+\frac{1}{2}\right) \alpha_{2 s}\left(f_{\mathcal{D}, \lambda} \cdot u_{\mathcal{D}, 0, t-s}\right), \\
& d_{t}(\lambda, \overline{0})=-2 w_{0}^{-1 / 2} \sum_{j=0}^{t}(-1)^{j-t}\left(\begin{array}{c}
t+1 \\
j+1
\end{array}\right) \sum_{s=0}^{j} \Gamma\left(s+\frac{1}{2}\right) \alpha_{2 s}\left(f_{\mathcal{D}, \lambda}^{*} \cdot u_{\mathcal{D}, \lambda, j-s}\right) .
\end{aligned}
$$

The asymptotic expansion of $\mathcal{E}_{1}(N,-\lambda N)$ is proved analogously to [O'S16b, Sect. 8]. The functions $f_{\mathcal{E}, \lambda}, \phi_{\lambda, k}^{*}$ and $u_{0, j}$ are defined in (6.36), (6.43) and (5.17) respectively. 


\begin{tabular}{cc|ccrr|r}
$N$ & $\lambda$ & $m=1$ & $m=2$ & \multicolumn{1}{c}{$m=3$} & \multicolumn{1}{c}{$m=5$} & \multicolumn{1}{c}{$\mathcal{D}_{1}(N,-\lambda N)$} \\
\hline 1200 & $1 / 3$ & $-1.54767 \times 10^{12}$ & $-1.53845 \times 10^{12}$ & $-1.53755 \times 10^{12}$ & $-1.53757 \times 10^{12}$ & $-1.53757 \times 10^{12}$ \\
1200 & 1 & $2.19568 \times 10^{12}$ & $2.20664 \times 10^{12}$ & $2.20164 \times 10^{12}$ & $2.20181 \times 10^{12}$ & $2.20181 \times 10^{12}$ \\
1200 & 2 & $-5.91241 \times 10^{12}$ & $-5.62009 \times 10^{12}$ & $-5.60529 \times 10^{12}$ & $-5.60869 \times 10^{12}$ & $-5.60866 \times 10^{12}$ \\
\hline 1203 & $1 / 3$ & $-2.17797 \times 10^{12}$ & $-2.18005 \times 10^{12}$ & $-2.17903 \times 10^{12}$ & $-2.17904 \times 10^{12}$ & $-2.17904 \times 10^{12}$ \\
1203 & 1 & $1.68218 \times 10^{12}$ & $1.74105 \times 10^{12}$ & $1.73537 \times 10^{12}$ & $1.73545 \times 10^{12}$ & $1.73545 \times 10^{12}$ \\
1203 & 2 & $-7.82854 \times 10^{12}$ & $-7.68896 \times 10^{12}$ & $-7.65629 \times 10^{12}$ & $-7.66011 \times 10^{12}$ & $-7.66007 \times 10^{12}$
\end{tabular}

Table 7: The approximations of Theorem 7.3 to $\mathcal{D}_{1}(N,-\lambda N)$.

Theorem 7.4. Let $\lambda^{+}$be a positive real number. Suppose $N \in \mathbb{Z}_{\geqslant 1}$ and $\lambda N \in \mathbb{Z}$ for $\lambda$ satisfying $|\lambda| \leqslant \lambda^{+}$. We have

$$
\mathcal{E}_{1}(N,-\lambda N)=\operatorname{Re}\left[\frac{w(0,-2)^{-N}}{N^{2}}\left(e_{0}(\lambda)+\frac{e_{1}(\lambda)}{N}+\cdots+\frac{e_{m-1}(\lambda)}{N^{m-1}}\right)\right]+O\left(\frac{|w(0,-2)|^{-N}}{N^{m+2}}\right)
$$

for an implied constant depending only on $\lambda^{+}$and $m$. The functions $e_{t}(\lambda)$ are given as follows, where $\alpha$ depends on $p$ with saddle-point $z_{1}$,

$$
e_{t}(\lambda)=2 \sum_{s=0}^{t} \sum_{k=0}^{t-s} \Gamma\left(s+\frac{1}{2}\right) \alpha_{2 s}\left(f_{\mathcal{E}, \lambda} \cdot \phi_{\lambda, k}^{*} \cdot u_{0, t-s-k}\right) .
$$

\begin{tabular}{cc|cccc|c}
$N$ & $\lambda$ & $m=1$ & $m=2$ & $m=3$ & $m=5$ & \multicolumn{1}{c}{$\mathcal{E}_{1}(N,-\lambda N)$} \\
\hline 1200 & $1 / 3$ & $1.14604 \times 10^{8}$ & $1.18408 \times 10^{8}$ & $1.17881 \times 10^{8}$ & $1.17905 \times 10^{8}$ & $1.17905 \times 10^{8}$ \\
1200 & 1 & $-2.26622 \times 10^{8}$ & $-2.83349 \times 10^{8}$ & $-2.67613 \times 10^{8}$ & $-2.69606 \times 10^{8}$ & $-2.69595 \times 10^{8}$ \\
1200 & 2 & $-1.25746 \times 10^{10}$ & $-9.18677 \times 10^{9}$ & $-9.62868 \times 10^{9}$ & $-9.62637 \times 10^{9}$ & $-9.63726 \times 10^{9}$
\end{tabular}

Table 8: The approximations of Theorem 7.4 to $\mathcal{E}_{1}(N,-\lambda N)$.

\section{When waves are good approximations to partitions}

\subsection{Proof of Theorem 1.4}

We first show upper and lower bounds for $p_{N}\left(\lambda N^{2}\right)$.

Lemma 8.1. For positive integers $N, \lambda N^{2}$ and absolute implied constants

$$
\frac{1}{\lambda N^{2}} e^{(2+\log \lambda) N} \ll p_{N}\left(\lambda N^{2}\right) \ll \frac{1}{\lambda N^{2}} e^{(2 \pi \sqrt{\lambda / 6}) N} .
$$

Proof. The upper bound follows from $p_{N}(n) \leqslant p(n)$ and $(1.9)$. For the lower bound we start with

$$
\frac{1}{N !}\left(\begin{array}{c}
N+n-1 \\
N-1
\end{array}\right) \leqslant p_{N}(n)
$$

from, for example, [Sze51, p. 86]. Stirling's formula implies $\Gamma(x) \sim \sqrt{2 \pi} x^{x-1 / 2} e^{-x}$ as $x \rightarrow \infty$, so we see that the left side of $(8.2)$ is

$$
\frac{\Gamma(N+n)}{\Gamma(N)^{2} \Gamma(n) \cdot n \cdot N} \gg \frac{(\lambda e)^{N}}{\lambda N^{2}}\left(1+\frac{1}{\lambda N}\right)^{N(1+\lambda N)}
$$

for $n=\lambda N^{2}$. Now use that $(1+1 / x)^{1+x} \geqslant e$ for $x>0$ to show

$$
\left(1+\frac{1}{\lambda N}\right)^{N(1+\lambda N)} \geqslant e^{N}
$$

and we have proved the lower bound. 
Proof of Theorem 1.4 With $n=\lambda N^{2}$ in (3.1) and (3.4), we have

$$
p_{N}\left(\lambda N^{2}\right)=\sum_{k=1}^{100} W_{k}\left(N, \lambda N^{2}\right)-\sum_{h / k} Q_{h k\left(-\lambda N^{2}\right)}(N)
$$

where the second sum is over all $h / k \in \mathcal{A}(N) \cup \mathcal{B}(101, N) \cup \mathcal{C}(N) \cup \mathcal{D}(N) \cup \mathcal{E}(N)$. With (5.7) and (5.8), the sum over $\mathcal{A}(N)$ is bounded by an absolute constant times the absolute value of

$$
e^{0.05 N}+\frac{2}{N^{1 / 2}} \operatorname{Im} \sum_{k: \hat{z} \in(1.01,1.49)} \frac{(-1)^{k}}{k^{2}} \exp (-N(p(\hat{z})+2 \pi i \lambda \hat{z}-\pi i / \hat{z})) q(\hat{z}) \exp (v(\hat{z} ; N, 0))
$$

where $\hat{z}=N / k$ as before. We know that $q(z) \exp (v(z ; N, 0)) \ll 1$ by (5.12) with $\lambda=0$. For $z=x \in \mathbb{R}$ and $\lambda \in \mathbb{R}$

$$
\operatorname{Re}[-p(z)-2 \pi i \lambda z+\pi i / z]=\operatorname{Re}[-p(z)]=\frac{\operatorname{Im}\left(\operatorname{Li}_{2}\left(e^{2 \pi i x}\right)\right)}{2 \pi x}=\frac{\mathrm{Cl}_{2}(2 \pi x)}{2 \pi x}
$$

and the right side of $(8.6)$ is bounded by 0.15 for $1 \leqslant x \leqslant 1.5$. Consequently, (8.5) is bounded by an absolute constant times

$$
e^{0.05 N}+e^{0.15 N} \sum_{k: \hat{z} \in(1.01,1.49)} \frac{1}{k^{2}} \ll e^{0.15 N} .
$$

Similar reasoning, using (6.13), 6.17), 6.29), 6.31) and 6.42), shows the sums over $\mathcal{C}(N), \mathcal{D}(N)$ and $\mathcal{E}(N)$ are also $O\left(e^{0.15 N}\right)$.

From the proof of Proposition 6.1 we find that the sum over $\mathcal{B}(101, N)$ is $O\left(e^{(|\lambda|+0.055) N}\right)$. Therefore

$$
p_{N}\left(\lambda N^{2}\right)=\sum_{k=1}^{100} W_{k}\left(N, \lambda N^{2}\right)+O\left(e^{0.15 N}+e^{(|\lambda|+0.055) N}\right) .
$$

Next, we divide both sides of (8.7) by $p_{N}\left(\lambda N^{2}\right)$ and use the lower bound of (8.1). Since

$$
\max \{0.15,|\lambda|+0.055\}-2-\log \lambda<-0.109
$$

for $0.2 \leqslant \lambda \leqslant 2.9$ we obtain

$$
p_{N}\left(\lambda N^{2}\right) \backslash\left(\sum_{k=1}^{100} W_{k}\left(N, \lambda N^{2}\right)\right)=1+O\left(e^{-0.1 N}\right)
$$

and the result follows easily.

\subsection{Good approximations for $N$ or $n$ small}

In Figure 2 we see how closely $W_{1}(n, n)$ matches $p(n)$ for relatively small $n$. We know that the first waves oscillate with periodicity of length $2 \pi / V \approx 31.96311$ for large $n$ and this periodicity is already visible in the figure. Our computations show, for example, that

$$
\left|1-\frac{W_{1}(n, n)}{p(n)}\right|<2 \times 10^{-6} \text { for } \quad 100 \leqslant n \leqslant 700 .
$$

The smallest value of $\left|1-W_{1}(n, n) / p(n)\right|$ for $1 \leqslant n \leqslant 700$ occurs when $n=432$, giving the very accurate approximation

$$
\begin{aligned}
W_{1}(432,432) & \approx \underline{4664786328422} 8241960.9, \\
p(432) & =\underline{4664786328422} 9267991
\end{aligned}
$$




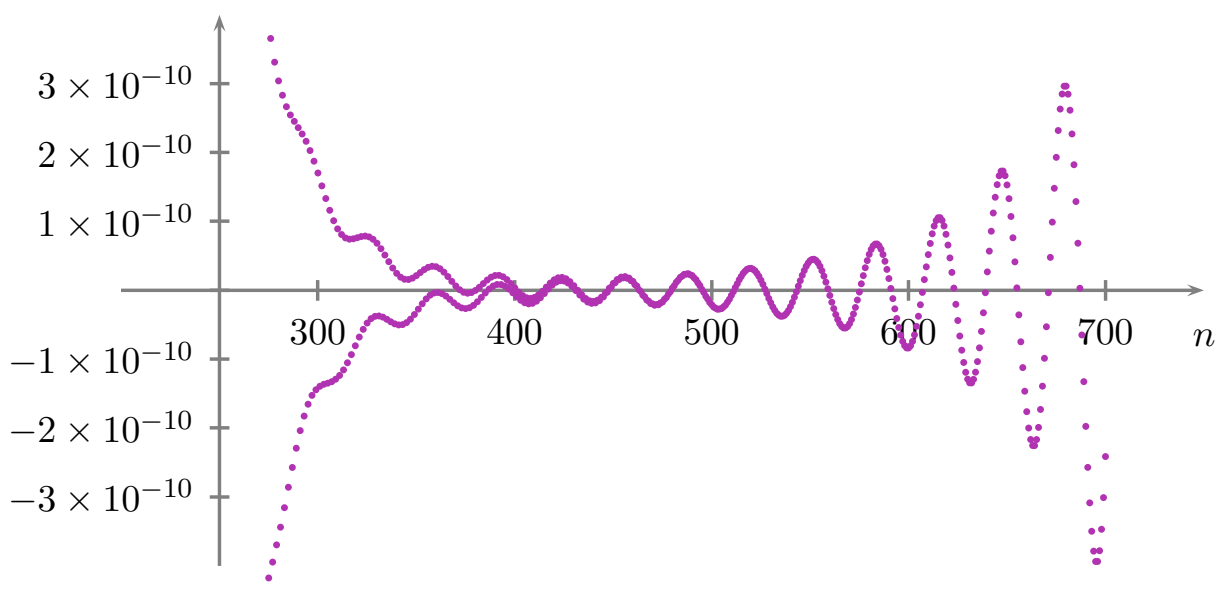

Figure 2: The function $1-W_{1}(n, n) / p(n)$ for $250 \leqslant n \leqslant 700$

For $\lambda=2$ the initial agreement between $p_{N}(2 N)$ and $W_{1}(N, 2 N)$ is even more impressive. For example,

$$
\left|1-\frac{W_{1}(N, 2 N)}{p_{N}(2 N)}\right| \approx 5.86 \times 10^{-21} \text { for } \quad N=1000 .
$$

For $N=2000$ it is $\approx 2.54 \times 10^{-12}$. We computed the restricted partition $p_{N}(2 N)$ above by relating it to $p(n)$ with the formula

$$
p_{N}(2 N)=p(2 N)-\sum_{m=0}^{N-1} p(m)
$$

This follows since we must remove from the partitions in $p(2 N)$ those with largest part of size $2 N-m$. The partition function $p(n)$ may be easily calculated using (1.8).

Examples of Theorem 1.2 with $\lambda=2$ are shown in Table 9 . This shows that after about $N=2900$ the first wave begins to grow more rapidly than $p_{N}(2 N)$ and follows the expected asymptotics. See also the bottom row in Table 3 for more on the $N=3300$ case.

\begin{tabular}{c|c|c|c}
$N$ & $p_{N}(2 N)$ & $W_{1}(N, 2 N)$ & $\operatorname{Re}\left[\left(2 z_{0} e^{-5 \pi i z_{0}}\right) \frac{w_{0}^{-N}}{N^{2}}\right]$ \\
\hline 2700 & $\mathbf{1 . 9 4} \times \mathbf{1 0}^{\mathbf{7 7}}$ & $\mathbf{1 . 9 2} \times \mathbf{1 0}^{\mathbf{7 7}}$ & $-1.16 \times 10^{75}$ \\
2800 & $\mathbf{5 . 9 3} \times \mathbf{1 0}^{\mathbf{7 8}}$ & $\mathbf{5 . 4 9} \times \mathbf{1 0}^{\mathbf{7 8}}$ & $-4.33 \times 10^{77}$ \\
2900 & $1.71 \times 10^{80}$ & $4.63 \times 10^{80}$ & $3.20 \times 10^{80}$ \\
3000 & $4.67 \times 10^{81}$ & $\mathbf{6 . 6 0} \times \mathbf{1 0}^{\mathbf{8 3}}$ & $\mathbf{6 . 8 3} \times \mathbf{1 0}^{\mathbf{8 3}}$ \\
3100 & $1.21 \times 10^{83}$ & $\mathbf{5 . 5 9} \times \mathbf{1 0}^{\mathbf{8 6}}$ & $\mathbf{5 . 7 0} \times \mathbf{1 0}^{\mathbf{8 6}}$ \\
3200 & $2.96 \times 10^{84}$ & $\mathbf{1 . 8 3} \times \mathbf{1 0}^{\mathbf{8 9}}$ & $\mathbf{1 . 7 8} \times \mathbf{1 0}^{\mathbf{8 9}}$ \\
3300 & $6.92 \times 10^{85}$ & $-\mathbf{1 . 8 9} \times \mathbf{1 0}^{\mathbf{9 2}}$ & $-\mathbf{2 . 0 3} \times \mathbf{1 0}^{\mathbf{9 2}}$
\end{tabular}

Table 9: Comparing $p_{N}(2 N), W_{1}(N, 2 N)$ and the asymptotics from Theorem 1.2 .

We can offer the following explanation for why there is such good initial agreement between $p_{N}(\lambda N)$ and the first waves. From (3.11) in the proof of Theorem 1.2 we know that

$$
\sum_{k=1}^{100} W_{k}(N, \lambda N)=p_{N}(\lambda N)+\frac{e^{U N}}{N^{2}} \psi_{\lambda} \cdot \sin \left(\tau_{\lambda}+V N\right)+O\left(\frac{e^{U N}}{N^{3}}\right),
$$

recalling (1.15), (1.18) and (1.19). For small $N, p_{N}(\lambda N)$ is the largest term on the right of (8.8). This means the first waves will be close to $p_{N}(\lambda N)$. However $p_{N}(\lambda N) \ll \exp ((2 \pi \sqrt{|\lambda| / 6}) \sqrt{N})$ as in (3.12) which implies that, for any given $\lambda$, the second term on the right of (8.8) will always eventually dominate and hence provide the asymptotics for the first waves as $N \rightarrow \infty$. 


\section{Future work}

As we noted in Section 1.2, the main results of Theorems 1.1, 1.2, 1.4 and Corollary 1.3 should be true with the sum over the first 100 waves replaced by just the first wave. Hence Theorem 1.2 becomes:

Conjecture 9.1. Let $\lambda^{+}$be a positive real number. Suppose $N \in \mathbb{Z}_{\geqslant 1}$ and $\lambda N \in \mathbb{Z}$ for $\lambda$ satisfying $|\lambda| \leqslant \lambda^{+}$. Then

$$
W_{1}(N, \lambda N)=\operatorname{Re}\left[\frac{w_{0}^{-N}}{N^{2}}\left(a_{0}(\lambda)+\frac{a_{1}(\lambda)}{N}+\cdots+\frac{a_{m-1}(\lambda)}{N^{m-1}}\right)\right]+O\left(\frac{\left|w_{0}\right|^{-N}}{N^{m+2}}\right)
$$

where the coefficients $a_{t}(\lambda)$ are given in (5.21) and the implied constant depends only on $\lambda^{+}$and $m$.

We have already seen evidence for this conjecture in Table 3, Its proof would require an improvement in Proposition 6.1, the techniques of [Sze51] or [DG14] might allow a careful enough estimate of $W_{2}+W_{3}+$ $\cdots+W_{100}$. Strengthening Proposition 6.1 would also increase the allowable range of $\lambda$ for Theorem 1.4

Numerical experiments reveal that $W_{2}(N, \lambda N)$ matches $(-1)^{N+1} \mathcal{D}_{1}(N,-\lambda N)$ for large $N$ and we expect their asymptotic expansions are the same:

Conjecture 9.2. Let $\lambda^{+}$be a positive real number. Suppose $N \in \mathbb{Z}_{\geqslant 1}$ and $\lambda N \in \mathbb{Z}$ for $\lambda$ satisfying $|\lambda| \leqslant \lambda^{+}$. Then with $\bar{N}$ denoting $N$ mod 2 , we have

$$
W_{2}(N, \lambda N)=(-1)^{N+1} \operatorname{Re}\left[\frac{w_{0}^{-N / 2}}{N^{2}}\left(d_{0}(\lambda, \bar{N})+\frac{d_{1}(\lambda, \bar{N})}{N}+\cdots+\frac{d_{m-1}(\lambda, \bar{N})}{N^{m-1}}\right)\right]+O\left(\frac{\left|w_{0}\right|^{-N / 2}}{N^{m+2}}\right)
$$

for an implied constant depending only on $\lambda^{+}$and $m$. The functions $d_{t}(\lambda, \bar{N})$ are given in (7.5) and (7.6).

\begin{tabular}{cc|cccc|c}
$N$ & $\lambda$ & $m=1$ & $m=2$ & $m=3$ & \multicolumn{1}{c}{$m=5$} & \multicolumn{1}{c}{$W_{2}(N, \lambda N)$} \\
\hline 3000 & $1 / 3$ & $6.13580 \times 10^{37}$ & $6.18769 \times 10^{37}$ & $6.18681 \times 10^{37}$ & $6.18680 \times 10^{37}$ & $6.18680 \times 10^{37}$ \\
3000 & 1 & $2.20860 \times 10^{36}$ & $-2.19459 \times 10^{35}$ & $-1.79624 \times 10^{35}$ & $-1.79070 \times 10^{35}$ & $-1.79070 \times 10^{35}$ \\
3000 & $5 / 3$ & $-1.84871 \times 10^{38}$ & $-1.77143 \times 10^{38}$ & $-1.77234 \times 10^{38}$ & $-1.77239 \times 10^{38}$ & $-1.77190 \times 10^{38}$
\end{tabular}

Table 10: The approximations of Conjecture 9.2 to $W_{2}(N, \lambda N)$.

Table 10 gives examples and the agreement for $N$ odd is similar. Conjecture 9.2 is the analog of [O'S16a, Conj. 6.4] for the Rademacher coefficients. Conjectures 9.1 and 9.2 together imply that $W_{2}(N, \lambda N)$ squared is approximately $W_{1}(N, \lambda N) / N^{2}$ for large $N$.

Comparing Tables 5 and 8 , we see that the entries for $\mathcal{E}_{1}(N,-\lambda N)$ in Table 8 are exactly 3 times the entries for $\mathcal{C}_{2}(N,-\lambda N)$ in Table 5 . The following is based on further numerical evidence.

Conjecture 9.3. The quantities $\mathcal{E}_{1}(N,-\lambda N)$ and $3 \mathcal{C}_{2}(N,-\lambda N)$, given by

$$
2 \operatorname{Re} \sum_{\frac{N}{3}<k \leqslant \frac{N}{2}} Q_{1 k(-\lambda N)}(N) \quad \text { and } \quad 6 \operatorname{Re} \sum_{\frac{2 N}{3}<k \leqslant N, k \text { odd }} Q_{2 k(-\lambda N)}(N)
$$

respectively, have the same asymptotic expansion as $N \rightarrow \infty$. In other words, recalling (7.2), (7.8), we have $e_{t}(\lambda)=3 c_{t}(\lambda)$ for all $t \in \mathbb{Z}_{\geqslant 0}$.

We may finally ask how our asymptotic results extend to other examples of the more general waves of Section 2.3 .

\section{References}

[Apo51] T. M. Apostol. On the Lerch zeta function. Pacific J. Math., 1:161-167, 1951.

[BDR02] Matthias Beck, Ricardo Diaz, and Sinai Robins. The Frobenius problem, rational polytopes, and FourierDedekind sums. J. Number Theory, 96(1):1-21, 2002. 
[BGK01] Matthias Beck, Ira M. Gessel, and Takao Komatsu. The polynomial part of a restricted partition function related to the Frobenius problem. Electron. J. Combin., 8(1):Note 7, 5 pp. (electronic), 2001.

[Cay56] Arthur Cayley. Researches on the partition of numbers. Philos. Trans. R. Soc. Lond., 146:127-140, 1856.

[CFW87] John A. Campbell, Per Olof Fröman, and Erik Walles. Explicit series formulae for the evaluation of integrals by the method of steepest descents. Stud. Appl. Math., 77(2):151-172, 1987.

[Com74] Louis Comtet. Advanced combinatorics. D. Reidel Publishing Co., Dordrecht, enlarged edition, 1974. The art of finite and infinite expansions.

[DG14] Michael Drmota and Stefan Gerhold. Disproof of a conjecture by Rademacher on partial fractions. Proc. Amer. Math. Soc. Ser. B, 1:121-134, 2014.

[Dowa] J. S. Dowker. On Sylvester waves and restricted partitions. arXiv: 1302.6172.

[Dowb] J. S. Dowker. Relations between the Ehrhart polynomial, the heat kernel and Sylvester waves. arXiv: 1108.1760 .

[DV17] Karl Dilcher and Christophe Vignat. An explicit form of the polynomial part of a restricted partition function. Res. Number Theory, 3:Art. 1, 12, 2017.

[FR02] Leonid G. Fel and Boris Y. Rubinstein. Sylvester waves in the Coxeter groups. Ramanujan J., 6(3):307-329, 2002.

[Gla09] J. W. L. Glaisher. Formulae for partitions into given elements, derived from Sylvester's theorem. Quart. J. Pure and Appl. Math., 40:275-348, 1909.

[HW08] G. H. Hardy and E. M. Wright. An introduction to the theory of numbers. Oxford University Press, Oxford, sixth edition, 2008. Revised by D. R. Heath-Brown and J. H. Silverman, With a foreword by Andrew Wiles.

[Max03] Leonard C. Maximon. The dilogarithm function for complex argument. R. Soc. Lond. Proc. Ser. A Math. Phys. Eng. Sci., 459(2039):2807-2819, 2003.

[O'S] Cormac O'Sullivan. Revisiting the saddle-point method of Perron. arXiv: 1702.03611.

[O'S15] Cormac O'Sullivan. On the partial fraction decomposition of the restricted partition generating function. Forum Math., 27(2):735-766, 2015.

[O'S16a] Cormac O'Sullivan. Asymptotics for the partial fractions of the restricted partition generating function I. Int. J. Number Theory, 12(6):1421-1474, 2016.

[O'S16b] Cormac O'Sullivan. Asymptotics for the partial fractions of the restricted partition generating function II. Integers, 16:Paper No. A78, 73 pp, 2016.

[O’S16c] Cormac O'Sullivan. Zeros of the dilogarithm. Math. Comp., 85(302):2967-2993, 2016.

[Per17] Oskar Perron. Über die näherungsweise Berechnung von Funktionen großer Zahlen. Sitzungsber. Bayr. Akad. Wissensch. (Münch. Ber.), pages 191-219, 1917.

[Rad73] Hans Rademacher. Topics in analytic number theory. Springer-Verlag, New York, 1973. Edited by E. Grosswald, J. Lehner and M. Newman, Die Grundlehren der mathematischen Wissenschaften, Band 169.

[RF06] Boris Y. Rubinstein and Leonid G. Fel. Restricted partition functions as Bernoulli and Eulerian polynomials of higher order. Ramanujan J., 11(3):331-347, 2006.

[Syl82] J. J. Sylvester. On subvariants, i.e. semi-invariants to binary quantics of an unlimited order: Excursus on rational fractions and partitions. Amer. J. Math., 5(1):119-136, 1882.

[SZ12] Andrew V. Sills and Doron Zeilberger. Formulæ for the number of partitions of $n$ into at most $m$ parts (using the quasi-polynomial ansatz). Adv. in Appl. Math., 48(5):640-645, 2012.

[Sze51] G. Szekeres. An asymptotic formula in the theory of partitions. Quart. J. Math., Oxford Ser. (2), 2:85-108, 1951.

[Zag07] Don Zagier. The dilogarithm function. In Frontiers in number theory, physics, and geometry. II, pages 3-65. Springer, Berlin, 2007.

Dept. of Math, The CUNy Graduate Center, 365 Fifth Avenue, New York, Ny 10016-4309, U.S.A. E-mail address: cosullivan@gc. cuny . edu 